Special Section on Transition Metal Catalyzed Synthesis of Medicinally Relevant Molecules

\title{
Copper (cat) and phenylboronic acid mediated deformylative C-N coupling of isoindolinone-3-ols with formamides provide $\mathbf{C}(3)$ aminoisoindolinones
}

\author{
H SURYA PRAKASH RAO*, J PRABHAKARAN, SILAMBARASAN KANNIYAPPAN and \\ A VEERA BHADRA RAO \\ Department of Chemistry, Pondicherry University, Puducherry 605 014, India \\ E-mail: hspr.che@pondiuni.edu.in
}

MS received 8 January 2018; revised 22 April 2018; accepted 25 April 2018; published online 14 June 2018

\begin{abstract}
Copper(I) iodide efficiently catalyzes deformylative C-N coupling of formamides and isoindolinone3 -ols to provide $\mathrm{C}(3)$ primary/secondary amine substituted isoindolinones. The transformation requires a stoichiometric amount of phenylboronic acid as a co-reactant. In the reaction, formamides act as the synthetic equivalent of primary/secondary amines. The method is amenable for the synthesis of a combinatorial library of medicinally relevant $\mathrm{C}(3)$ amino substituted isoindolinones.
\end{abstract}

Keywords. Isoindolinones; copper catalysis; deformylative C-N coupling; phenylboronic acid.

\section{Introduction}

Isoindole is not as ubiquitous as indole, it is, however, represented as a part-structure in many bio-active alkaloids. ${ }^{1}$ Isoindolinones (1,3-dihydro- $2 \mathrm{H}$-isoindole1-one; phthalimidines; the highlighted portion in stachybotrysam A 1, Figure 1) form a distinctive sub-group among isoindoles. Significantly, several alkaloids isolated from marine fungi (e.g., 1) imbibe isoindole core. ${ }^{2}$ Alkaloids with this heterocyclic core exhibit potent and varied biological activities and thus isoindole is considered as a medicinally privileged structure. ${ }^{3}$ For example, the potent antifungal agent pestalachloride A 2 (Figure 1), isolated from the fungus Pestalotiopsis adusta has an isoindolinone core. ${ }^{4}$ Over the past few years, medicinal chemists have been engaged in the synthesis and biological evaluation of a variety heterocyclic compounds with this core; with an aim to unearth pharmaceutical compounds. ${ }^{5}$ Such efforts yielded development of anticancer compound lenalidomide $\mathbf{3}$, used for treating multiple myeloma, ${ }^{6}$ and $(S)$-pazinaclone $\mathbf{4}$ and its siblings used for sedative and anxiolytic effects. ${ }^{7}$ Among the family of isoindolinone heterocyclic compounds, synthesis of those which have a substitution on
$\mathrm{N}(2)$ and $\mathrm{C}(3)$ have attracted greater attention (highlighted portion in pazinaclone 4, Figure 1). ${ }^{8}$ Several efforts were directed towards the synthesis of this core structure, most of which go through cyclization to form an isoindolinone nucleus in the final step. ${ }^{9}$

For the past few years, we have been engaged in the copper-mediated synthesis of a variety of heterocyclic compounds via $\mathrm{C}\left(\mathrm{sp}^{3}\right)-\mathrm{C}\left(\mathrm{sp}^{2}\right)$ coupling reactions. ${ }^{10}$ In the context of the synthesis of $\mathrm{C}(3)$ substituted isoindolinones, we have reported a facile coppermediated coupling of 3-hydoxyisoindolinones $\mathbf{5}$ and aryl-, heteroaryl- and alkenylboronic acids with concomitant removal of boric acid to provide C(3) aryl/ heteroaryl/alkenyl substituted isoindolinones 6 (Equation 1, Scheme 1). ${ }^{11}$ Among the various copper catalysts, tried copper(II) trifluoromethanesulfonate $\left(\mathrm{Cu}(\mathrm{OTf})_{2}\right)$ worked well in dichloroethane (DCE) reflux. Both inter- and intra-molecular versions of the reaction were facile. When we conducted the reaction of $\mathbf{5}$ in $N, N$-dimethylformamide (DMF) medium, unexpectedly, it provided C-N coupled product 7 instead of anticipated C-C coupled product 6 (Equation 2, Scheme 1). The product 7 , obviously did not have the component of phenylboronic acid, but the reaction did

\footnotetext{
*For correspondence

Electronic supplementary material: The online version of this article (https://doi.org/10.1007/s12039-018-1474-8) contains supplementary material, which is available to authorized users.
} 


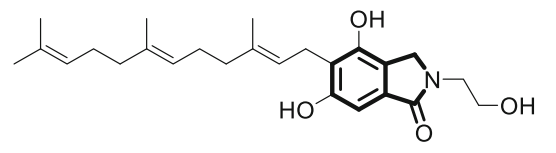

1 (Stachybotrysam A)

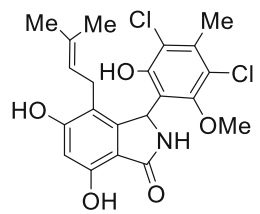

2 (Pestalachloride A)

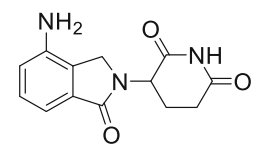

3 (Lenalidomide)

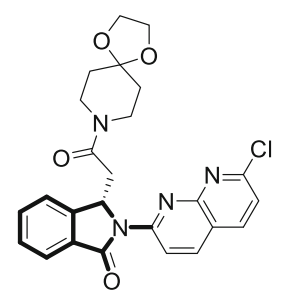

4 ((S)-Pazinaclone)

Figure 1. Representative examples of natural products (1 and 2) and synthetic drugs (3 and $\mathbf{4})$ with isoindolinone core.

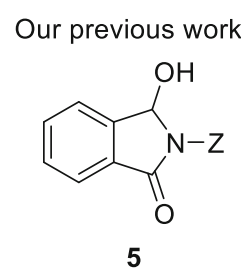

5

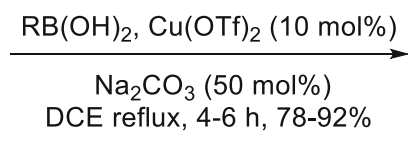

DCE reflux, 4-6 h, 78-92\%

$\mathrm{R}=$ aryl, heteroaryl, alkenyl; $\mathrm{Z}=\mathrm{C}_{6} \mathrm{H}_{5} \mathrm{CH}_{2}, 4-\mathrm{MeOC}_{6} \mathrm{H}_{4} \mathrm{CH}_{2}, 2-\mathrm{NO}_{2} \mathrm{C}_{6} \mathrm{H}_{4} \mathrm{CH}_{2}$
(Equation 1)

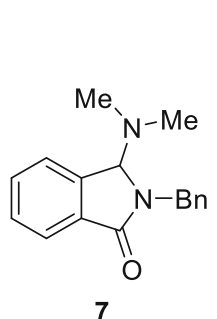

7

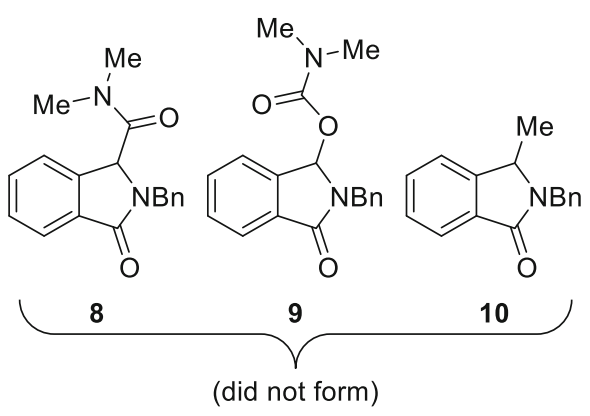

(Equation 2)

Scheme 1. Our previous and present work on the synthesis of C(3) substituted isoindolinones 6 and 7.

not take place in the absence of this reagent as well as in the absence of copper catalyst. The product formation of 7 was evidently going through deformylation of DMF. Since the reaction was going to completion only in the presence of one equivalent of phenylboronic acid, we concluded that it was a co-reactant. Overall, the intriguing and surprising result prompted us to explore the reaction in greater detail and the results are reported herein. Of late, copper-catalyzed transformations are gaining more popularity over palladium catalyzed reactions as the copper catalysts are less expensive, less toxic and biocompatible. ${ }^{12}$ Recently, Palombi and coworkers reported access to isoindolinones of the type 7 ( $N$-Mannich bases of 1 -isoindolinones) by the reaction of 2-formylbenzonitriles with primary or secondary amines under chemical or electrochemical activation. ${ }^{13}$ In our reaction, DMF (Equation 2, Scheme 1) is a surrogate and convenient source of dimethyl amine which is a toxic gas at $\mathrm{rt}\left(\right.$ B.p. $\left.=7^{\circ} \mathrm{C}\right)$. Previously, DMF and similar formamides have been used as a source of (i) $N$-acylamines, (ii) amines, (iii) alkyl groups to effect a variety of coupling reactions conducted under copper catalysis under aerobic/oxidative conditions. ${ }^{14}$ In our reaction, deformylataive $\mathrm{C}-\mathrm{N}$ coupled product 7 formed exclusively. The reaction did not provide even traces of dehydrative C-C coupled product $\mathbf{8}$, dehydrogenative C-O coupled product $\mathbf{9}$ or methylated product $\mathbf{1 0}$ (Scheme 1). To the best of our knowledge, copper and phenylboronic acid-mediated deformylative and dehydroxylative $\mathrm{C}-\mathrm{N}$ coupling as seen in the conversion of 3-hydoxyisoindolinone $\mathbf{5}$ to $\mathrm{C}(3) \mathrm{N}, \mathrm{N}$-dimethylamino isoindolinone $\mathbf{7}$ is not known and is being reported for the first time.

\section{Experimental}

\subsection{General Experimental Methods}

The progress of all the reactions was monitored by TLC using a suitable mixture of hexanes (a mixture of aliphatic hydrocarbons that boil between $60-80^{\circ} \mathrm{C}$ ) and ethyl acetate as an 
eluent. Column chromatography was performed on silica gel (100-200 mesh) using increasing percentages of ethyl acetate in hexanes. ${ }^{1} \mathrm{H}$ NMR (400 MHz), ${ }^{13} \mathrm{C}$ NMR $(100 \mathrm{MHz})$ and DEPT-135 spectra were recorded for $\mathrm{CDCl}_{3}$ or $\mathrm{CDCl}_{3} / \mathrm{CCl}_{4}$ (1/1) solutions with a $400 \mathrm{MHz}$ NMR spectrometer with TMS as the internal standard. Chemical shifts (in ppm) and coupling constants $(J$ in $\mathrm{Hz}$ ) are given here. IR spectra were recorded as $\mathrm{KBr}$ pellets with a Nicolet-6700 FT-IR spectrometer. The melting points were recorded with open-ended capillary tubes with a VEEGO VMP-DS instrument and are uncorrected. High-resolution mass spectra were recorded on a quadrupole time-of-flight (QTOF) mass spectrometer using electro-spray ionization mode (ESIMS). X-Ray diffraction measurements were carried out at $298 \mathrm{~K}$ on a diffractometer equipped with a graphite monochromator and a Mo-K $\alpha$ fine focus sealed tube $(\lambda=0.71073 \AA)$. Organic solvents were dried by standard methods. ${ }^{15}$ Commercially obtained reagents were used after purification. Arylboronic acids were purchased from Sigma-Aldrich and copper salts were purchased from Avra Chemicals.

\subsection{General Procedure for synthesis of $N(2)$ substituted C(3) aminoisoindolin-1-ones Synthesis of 2-benzyl-3-(dimethylamino)isoindolin-1-one 7a}

To an oven-dried test tube charged with a mixture of 2benzyl-3-hydroxyisoindolin-1-one 5a (100 mg, 1 mmol,), $N, N$-dimethylformamide (DMF, $30 \mathrm{mg}, 1 \mathrm{mmol}$ ), copper iodide $(7.9 \mathrm{mg}, 0.1 \mathrm{mmol})$, phenylboronic acid $(50 \mathrm{mg}, 1$ mmol), $N$-methylpyrrolidinone $(1 \mathrm{~mL})$ were added. The test tube with the resulting inhomogeneous solution was kept in a pre-heated $\left(100^{\circ} \mathrm{C}\right)$ oil-bath and the contents were stirred. The progress of the reaction was periodically checked by TLC for its completion. After the disappearance of the reactants the reaction mixture was cooled to $\mathrm{rt}$ and then diluted with dichloromethane (DCM, $20 \mathrm{~mL})$ and water $(20 \mathrm{~mL})$. The aqueous layer was extracted with (DCM, $20 \mathrm{~mL}$ ). The organic layers were mixed and washed with water $(2 \times 20 \mathrm{~mL})$ and brine $(1 \times 10 \mathrm{~mL})$ sequentially. The organic layer was dried over $\mathrm{Na}_{2} \mathrm{SO}_{4}$. Removal of DCM by under reduced pressure resulted in a crude product which was purified by column chromatography (silica gel, gradient elution with increasing volume of EtOAc in hexanes) to yield pure 2benzyl-3-(dimethylamino)isoindolin-1-one as yellow solid.

\section{2a 2-Benzyl-3-(dimethylamino)isoindolin-1-one}

(7a):<smiles>CN(C)C1c2ccccc2C(=O)N1Cc1ccccc1</smiles>

Yellow solid (92 mg, $80 \%$ yield); M.p. $105^{\circ} \mathrm{C}$; IR ( $\mathrm{KBr}$, $\left.\mathrm{cm}^{-1}\right) 3343,3035,2926,2846,2787,1690,1425,1358,1279$, $1210,1124,1059,976,936,796,747,700,600,523 ;{ }^{1} \mathrm{H}$ NMR
(400 MHz, $\left.\mathrm{CDCl}_{3} / \mathrm{CCl}_{4}, 1 / 1\right) \delta 7.86-7.81$ (m, 1H), 7.44 (dd, $J=5.7,2.5 \mathrm{~Hz}, 2 \mathrm{H}), 7.40(\mathrm{~d}, J=6.4 \mathrm{~Hz}, 1 \mathrm{H}), 7.27(\mathrm{~d}$, $J=6.0 \mathrm{~Hz}, 2 \mathrm{H}), 7.26-7.22(\mathrm{~m}, 2 \mathrm{H}), 7.19(\mathrm{dd}, J=5.8$, $2.7 \mathrm{~Hz}, 1 \mathrm{H}), 5.21(\mathrm{~d}, J=14.7 \mathrm{~Hz}, 1 \mathrm{H}), 4.98$ (s, 1H), 4.21 $(\mathrm{d}, J=14.6 \mathrm{~Hz}, 1 \mathrm{H}), 2.26(\mathrm{~s}, 6 \mathrm{H}) ;{ }^{13} \mathrm{C} \mathrm{NMR}(100 \mathrm{MHz}$, $\left.\mathrm{CDCl}_{3} / \mathrm{CCl}_{4}, 1 / 1\right) \delta 167.3(\mathrm{C}), 142.2$ (C), 137.5 (C), 133.0 (C), $131.3(\mathrm{CH}), 129.1(\mathrm{CH}), 128.7(\mathrm{CH}), 128.5(\mathrm{CH}), 127.5$ $(\mathrm{CH}), 124.0,(\mathrm{CH}) 123.5(\mathrm{CH}), 77.3(\mathrm{CH}), 43.6\left(\mathrm{CH}_{2}\right), 39.1$ (2 x CH 3$)$; HRMS (ESI) calcd.. for $\mathrm{C}_{17} \mathrm{H}_{19} \mathrm{~N}_{2} \mathrm{O}(\mathrm{M}+\mathrm{H})$ 267.1497, found 267.1511.

\section{2b 2-Benzyl-3-(phenylamino)isoindolin-1-one (7b):}<smiles>O=C1c2ccccc2C(Nc2ccccc2)N1Cc1ccccc1</smiles>

White solid (103 mg, 78\% yield); M.p. $163^{\circ} \mathrm{C}$; IR $(\mathrm{KBr}$, $\mathrm{cm}^{-1}$ ) 3349, 3033, 2924, 2859, 1683, 1600, 1499, 1261, 1077, 1024, 805, 746, 698; ${ }^{1} \mathrm{H} \mathrm{NMR}\left(400 \mathrm{MHz}, \mathrm{CDCl}_{3} / \mathrm{CCl}_{4}, 1 / 1\right)$ $\delta 7.80(\mathrm{dd}, J=6.6,1.4 \mathrm{~Hz}, 1 \mathrm{H}), 7.50-7.44(\mathrm{~m}, 2 \mathrm{H}), 7.44$ $7.41(\mathrm{~m}, 1 \mathrm{H}), 7.25(\mathrm{~d}, J=1.7 \mathrm{~Hz}, 1 \mathrm{H}), 7.24(\mathrm{~d}, J=0.7 \mathrm{~Hz}$, $1 \mathrm{H}), 7.22(\mathrm{~d}, J=3.7 \mathrm{~Hz}, 1 \mathrm{H}), 7.16-7.13(\mathrm{~m}, 2 \mathrm{H}), 7.11(\mathrm{~d}$, $J=1.1 \mathrm{~Hz}, 1 \mathrm{H}), 7.11-7.08(\mathrm{~m}, 1 \mathrm{H}), 6.77(\mathrm{t}, J=7.4 \mathrm{~Hz}$, $1 \mathrm{H}), 6.55(\mathrm{dd}, J=8.5,0.9 \mathrm{~Hz}, 2 \mathrm{H}), 5.81(\mathrm{~s}, 1 \mathrm{H}), 5.11(\mathrm{~d}$, $J=15.1 \mathrm{~Hz}, 1 \mathrm{H}), 4.13(\mathrm{~d}, J=15.1 \mathrm{~Hz}, 1 \mathrm{H}) ;{ }^{13} \mathrm{C} \mathrm{NMR}(100$ $\left.\mathrm{MHz}, \mathrm{CDCl}_{3} / \mathrm{CCl}_{4}, 1 / 1\right) \delta 167.3(\mathrm{C}), 145.6(\mathrm{C}), 144.0(\mathrm{C})$, $137.4(\mathrm{C}), 132.19(\mathrm{C}), 132.12(\mathrm{CH}), 129.6(\mathrm{CH}), 129.5(\mathrm{CH})$, $128.7(\mathrm{CH}), 128.3(\mathrm{CH}), 127.6(\mathrm{CH}), 123.8(\mathrm{CH}), 123.2(\mathrm{CH})$, $119.5(\mathrm{CH}), 114.4(\mathrm{CH}), 68.8(\mathrm{CH}), 43.2\left(\mathrm{CH}_{2}\right)$; HRMS (ESI) calcd.. for $\mathrm{C}_{21} \mathrm{H}_{19} \mathrm{~N}_{2} \mathrm{O}(\mathrm{M}+\mathrm{H}) 315.1497$, found 315.1494 .

\section{2c 2-Benzyl-3-(cyclohexylamino)isoindolin-1-one}

(7c):

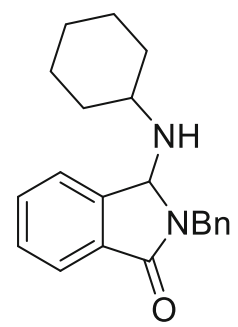

White solid (103 mg, 77\% yield); M.p. $104{ }^{\circ} \mathrm{C}$; IR ( $\mathrm{KBr}$, $\left.\mathrm{cm}^{-1}\right)$; 3347, 2928, 2845, 1670, 1510, 1467, 1391, 1354, 1306, 1235, 1176, 1121, 1090, 1035, 964, 842, 746, 694, 624, $520 ;{ }^{1} \mathrm{H} \mathrm{NMR}\left(400 \mathrm{MHz}, \mathrm{CDCl}_{3} / \mathrm{CCl}_{4}, 1 / 1\right) \delta 7.84-7.82(\mathrm{~m}$, $1 \mathrm{H}), 7.49-7.47$ (m, 2H), $7.44(\mathrm{dd}, J=8.8,2.1 \mathrm{~Hz}, 1 \mathrm{H}), 7.28$ (s, 2H), 7.27 (s, 2H), $7.20(\mathrm{dd}, J=8.6,4.5 \mathrm{~Hz}, 1 \mathrm{H}), 5.22(\mathrm{~d}$, $J=15.0 \mathrm{~Hz}, 1 \mathrm{H}), 5.16(\mathrm{~s}, 1 \mathrm{H}), 4.29(\mathrm{~d}, J=15.0 \mathrm{~Hz}, 1 \mathrm{H})$, $2.43(\mathrm{dd}, J=8.6,4.7 \mathrm{~Hz}, 1 \mathrm{H}), 1.68-1.63(\mathrm{~m}, 2 \mathrm{H}), 1.60$ $1.56(\mathrm{~m}, 1 \mathrm{H}), 1.50(\mathrm{~d}, J=4.4 \mathrm{~Hz}, 1 \mathrm{H}), 1.48-1.43(\mathrm{~m}, 1 \mathrm{H})$, $1.12(\mathrm{~d}, J=8.0 \mathrm{~Hz}, 2 \mathrm{H}), 1.09-1.02(\mathrm{~m}, 2 \mathrm{H}), 1.00-0.92$ 
(m, 1H); ${ }^{13} \mathrm{C}$ NMR (100 MHz, $\left.\mathrm{CDCl}_{3} / \mathrm{CCl}_{4}, 1 / 1\right) \delta 167.4$ (C), $145.1(\mathrm{C}), 137.7(\mathrm{C}), 132.4(\mathrm{C}), 131.6(\mathrm{CH}), 128.9(\mathrm{CH})$, $128.7(\mathrm{CH}), 128.2(\mathrm{CH}), 127.5(\mathrm{CH}), 123.6(\mathrm{CH}), 123.3(\mathrm{CH})$, $71.2(\mathrm{CH}), 51.9(\mathrm{CH}), 43.0\left(\mathrm{CH}_{2}\right), 35.3\left(\mathrm{CH}_{2}\right), 35.0\left(\mathrm{CH}_{2}\right)$, $25.8\left(\mathrm{CH}_{2}\right), 25.0\left(\mathrm{CH}_{2}\right), 24.93\left(\mathrm{CH}_{2}\right)$; HRMS (ESI) calcd. for $\mathrm{C}_{21} \mathrm{H}_{25} \mathrm{~N}_{2} \mathrm{O}(\mathrm{M}+\mathrm{H}) 321.1967$, found 321.1968 .

\section{2d 2-Benzyl-3-(benzylamino)isoindolin-1-one (7d):}<smiles>O=C1c2ccccc2C(NCc2ccccc2)N1Cc1ccccc1</smiles>

Yellow liquid (110 mg, 80\% yield); IR $\left(\mathrm{KBr}, \mathrm{cm}^{-1}\right)$; 3331, 3032, 2914, 1762, 1689, 1461, 1404, 1361, 1300, 1210, 1139, 1078, 966, 794, 741, 701, 519; ${ }^{1} \mathrm{H}$ NMR (400 MHz, $\left.\mathrm{CDCl}_{3}\right) \delta$ $7.85(\mathrm{~d}, J=7.3 \mathrm{~Hz}, 1 \mathrm{H}), 7.50(\mathrm{~d}, J=0.9 \mathrm{~Hz}, 1 \mathrm{H}), 7.49$ (s, 1H), 7.46-7.41 (m, 1H), $7.32(\mathrm{dd}, J=8.0,1.2 \mathrm{~Hz}, 2 \mathrm{H})$, $7.28(\mathrm{~d}, J=1.6 \mathrm{~Hz}, 1 \mathrm{H}), 7.27-7.24(\mathrm{~m}, 2 \mathrm{H}), 7.23(\mathrm{~s}, 1 \mathrm{H})$, $7.21(\mathrm{~d}, J=0.7 \mathrm{~Hz}, 1 \mathrm{H}), 7.18(\mathrm{~d}, J=7.0 \mathrm{~Hz}, 1 \mathrm{H}), 7.15-$ $7.10(\mathrm{~m}, 2 \mathrm{H}), 5.29(\mathrm{~s}, 1 \mathrm{H}), 5.11(\mathrm{~d}, J=15.0 \mathrm{~Hz}, 1 \mathrm{H}), 4.35$ $(\mathrm{d}, J=15.0 \mathrm{~Hz}, 1 \mathrm{H}), 3.31(\mathrm{~d}, J=13.1 \mathrm{~Hz}, 1 \mathrm{H}), 3.21(\mathrm{~d}$, $J=13.1 \mathrm{~Hz}, 1 \mathrm{H}), 2.28(\mathrm{~d}, J=7.6 \mathrm{~Hz}, 1 \mathrm{H}) ;{ }^{13} \mathrm{C}$ NMR $(100$ $\left.\mathrm{MHz}, \mathrm{CDCl}_{3}\right) \delta 167.7(\mathrm{C}), 143.4(\mathrm{C}), 139.7(\mathrm{C}), 137.5(\mathrm{C})$, $132.8(\mathrm{C}), 131.9(\mathrm{CH}), 129.1(\mathrm{CH}), 128.8(\mathrm{CH}), 128.4(\mathrm{CH})$, $128.3(\mathrm{CH}), 128.1(\mathrm{CH}), 127.6(\mathrm{CH}), 127.1(\mathrm{CH}), 123.4(\mathrm{CH})$, $123.3(\mathrm{CH}), 72.4(\mathrm{CH}), 45.5\left(\mathrm{CH}_{2}\right), 43.2\left(\mathrm{CH}_{2}\right)$; HRMS (ESI) calcd. for $\mathrm{C}_{22} \mathrm{H}_{21} \mathrm{~N}_{2} \mathrm{O}(\mathrm{M}+\mathrm{H}) 329.1654$, found 329.1648 .

\section{2e 2-Benzyl-3-(phenethylamino)isoindolin-1-one}

(7e):

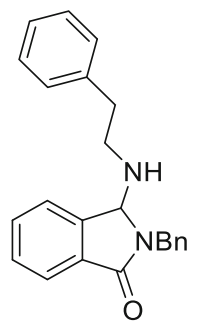

White solid (108 mg, 75\% yield); M.p. $105^{\circ} \mathrm{C}$; IR ( $\mathrm{KBr}$, $\mathrm{cm}^{-1}$ ) 3335, 3029, 2924, 2856, 1762, 1685, 1459, 1407, 1360, 1141, 1080, 1020, 745, 700, 557; ${ }^{1} \mathrm{H}$ NMR $(400 \mathrm{MHz}$, $\left.\mathrm{CDCl}_{3} / \mathrm{CCl}_{4}, 1 / 1\right) \delta 7.71(\mathrm{~d}, J=7.2 \mathrm{~Hz}, 1 \mathrm{H}), 7.34(\mathrm{dd}$, $J=7.4,1.3 \mathrm{~Hz}, 1 \mathrm{H}), 7.31(\mathrm{dd}, J=7.3,1.3 \mathrm{~Hz}, 1 \mathrm{H}), 7.25$ $(\mathrm{d}, J=7.1 \mathrm{~Hz}, 1 \mathrm{H}), 7.15(\mathrm{dd}, J=11.9,4.5 \mathrm{~Hz}, 2 \mathrm{H}), 7.09$ $(\mathrm{d}, J=6.7 \mathrm{~Hz}, 4 \mathrm{H}), 6.97(\mathrm{~d}, J=7.0 \mathrm{~Hz}, 4 \mathrm{H}), 5.00(\mathrm{~s}$, $1 \mathrm{H}), 4.95(\mathrm{~d}, J=14.9 \mathrm{~Hz}, 1 \mathrm{H}), 3.76(\mathrm{~d}, J=14.9 \mathrm{~Hz}, 1 \mathrm{H})$, $2.57-2.48(\mathrm{~m}, 1 \mathrm{H}), 2.45-2.36(\mathrm{~m}, 1 \mathrm{H}), 2.35-2.27(\mathrm{~m}, 1 \mathrm{H})$, $2.17(\mathrm{dt}, J=11.8,6.1 \mathrm{~Hz}, 1 \mathrm{H}), 2.02-1.86(\mathrm{~m}, 1 \mathrm{H}) ;{ }^{13} \mathrm{C}$
NMR (100 MHz, $\left.\mathrm{CDCl}_{3} / \mathrm{CCl}_{4}, 1 / 1\right) \delta 167.3(\mathrm{C}), 143.2(\mathrm{C})$, $139.5(\mathrm{C}), 137.1(\mathrm{C}), 132.7(\mathrm{C}), 131.5(\mathrm{CH}), 128.8(\mathrm{CH}), 128.7$ $(\mathrm{CH}), 128.6(\mathrm{CH}), 128.4(\mathrm{CH}), 128.0(\mathrm{CH}), 127.3(\mathrm{CH}), 126.3$ $(\mathrm{CH}), 123.3(\mathrm{CH}), 123.0(\mathrm{CH}), 71.8(\mathrm{CH}), 42.6\left(\mathrm{CH}_{2}\right), 41.7$ $\left(\mathrm{CH}_{2}\right), 36.0\left(\mathrm{CH}_{2}\right)$; HRMS (ESI) calcd. for $\mathrm{C}_{23} \mathrm{H}_{23} \mathrm{~N}_{2} \mathrm{O}(\mathrm{M}$ $+\mathrm{H}) 343.1810$, found 343.1847 .

$2.2 \mathrm{f} \quad 3-($ Benzo[d]thiazol-2-ylamino $)-2-$ benzylisoindolin-1-one (7f):<smiles>O=C1c2ccccc2C(Nc2nc3ccccc3s2)N1Cc1ccccc1</smiles>

White solid (125 mg, $80 \%$ yield); M.p. $170{ }^{\circ} \mathrm{C}$; IR ( $\mathrm{KBr}$, $\left.\mathrm{cm}^{-1}\right)$ 3256, 3037, 2926, 1754, 1693, 1603, 1538, 1443, 1261, 1208, 1127, 1065, 804, 749, 700; ${ }^{1} \mathrm{H}$ NMR (400 $\left.\mathrm{MHz}, \mathrm{CDCl}_{3} / \mathrm{CCl}_{4}, 1 / 1\right) \delta 7.77(\mathrm{dd}, J=6.7,1.0 \mathrm{~Hz}, 1 \mathrm{H})$, 7.61-7.55 (m, 2H), 7.54-7.45 (m, 3H), 7.35-7.26 (m, 3H), 7.24-7.19 (m, 3H), 7.17-7.11 (m, 1H), $6.48(\mathrm{~s}, 1 \mathrm{H}), 4.92(\mathrm{~d}$, $J=14.9 \mathrm{~Hz}, 1 \mathrm{H}), 4.28(\mathrm{~d}, J=14.9 \mathrm{~Hz}, 1 \mathrm{H}),{ }^{13} \mathrm{C}$ NMR $(100$ $\left.\mathrm{MHz}, \mathrm{CDCl}_{3} / \mathrm{CCl}_{4}, 1 / 1\right) \delta 167.6(\mathrm{C}), 165.3(\mathrm{C}), 151.6(\mathrm{C})$, $142.6(\mathrm{C}), 137.1(\mathrm{C}), 132.5(\mathrm{C}), 132.1(\mathrm{C}), 131.1(\mathrm{CH}), 130.0$ $(\mathrm{CH}), 128.7(\mathrm{CH}), 128.7(\mathrm{CH}), 127.8(\mathrm{CH}), 126.3(\mathrm{CH}), 123.8$ $(\mathrm{CH}), 123.6(\mathrm{CH}), 122.6(\mathrm{CH}), 121.0(\mathrm{CH}), 119.7(\mathrm{CH}), 68.4$ $(\mathrm{CH}), 43.8\left(\mathrm{CH}_{2}\right)$; HRMS (ESI) calcd. for $\mathrm{C}_{22} \mathrm{H}_{18} \mathrm{~N}_{3} \mathrm{OS}(\mathrm{M}$ + H) 372.1171 , found 372.1162 .

\section{2g 2-Benzyl-3-(piperidin-1-yl)isoindolin-1-one (7g):}<smiles>O=C1c2ccccc2C(N2CCCCC2)N1Cc1ccccc1</smiles>

Yellow solid (104 mg, 81\% yield); M.p. $115^{\circ} \mathrm{C}$; IR (KBr, $\left.\mathrm{cm}^{-1}\right)$ 3422, 2934, 2803, 1677, 1623, 1594, 1399, 1354, 1121, 1081, 986, 862, 750, 698, 631, 511; ${ }^{1} \mathrm{H}$ NMR $(400 \mathrm{MHz}$, $\left.\mathrm{CDCl}_{3} / \mathrm{CCl}_{4}, 1 / 1\right) \delta 7.85-7.79(\mathrm{~m}, 1 \mathrm{H}), 7.48-7.34(\mathrm{~m}, 3 \mathrm{H})$, $7.29(\mathrm{t}, J=5.5 \mathrm{~Hz}, 3 \mathrm{H}), 7.25-7.10(\mathrm{~m}, 2 \mathrm{H}), 5.17(\mathrm{~d}, J=$ $14.4 \mathrm{~Hz}, 1 \mathrm{H}), 4.90(\mathrm{~s}, 1 \mathrm{H}), 4.25$ (d, $J=13.6 \mathrm{~Hz}, 1 \mathrm{H}), 2.54$ (s, 2H), 2.37 (s, 2H), $1.52(\mathrm{~s}, 4 \mathrm{H}), 1.44(\mathrm{~s}, 2 \mathrm{H}) ;{ }^{13} \mathrm{C} \mathrm{NMR}(100$ $\left.\mathrm{MHz}, \mathrm{CDCl}_{3} / \mathrm{CCl}_{4}, 1 / 1\right) \delta 167.2(\mathrm{C}), 142.8(\mathrm{C}), 137.8$ (C), $133.1(\mathrm{C}), 131.1(\mathrm{CH}), 128.7(\mathrm{CH}), 128.7(\mathrm{CH}), 127.4(\mathrm{CH})$, $124.0(\mathrm{CH}), 123.4(\mathrm{CH}), 115.6(\mathrm{CH}), 77.9(\mathrm{CH}), 48.6\left(\mathrm{CH}_{2}\right)$, $43.2\left(\mathrm{CH}_{2}\right), 26.5\left(\mathrm{CH}_{2}\right), 24.6\left(\mathrm{CH}_{2}\right)$; HRMS (ESI) calcd. for $\mathrm{C}_{20} \mathrm{H}_{23} \mathrm{~N}_{2} \mathrm{O}(\mathrm{M}+\mathrm{H})$ 307.1810, found 307.1823. 
2.2h 2-Benzyl-3-morpholinoisoindolin-1-one (7h):<smiles>O=C1c2ccccc2C(N2CCOCC2)N1Cc1ccccc1</smiles>

White solid (94 mg, $73 \%$ yield); M.p. $129^{\circ} \mathrm{C}$; IR ( $\mathrm{KBr}$, $\left.\mathrm{cm}^{-1}\right)$ 3217, 2924, 2703, 1670, 1438, 1306, 1211, 1115, 1057, 969, 919, 743, 698, 633, 600, 519; ${ }^{1} \mathrm{H}$ NMR (400 $\left.\mathrm{MHz}, \mathrm{CDCl}_{3} / \mathrm{CCl}_{4}, 1 / 1\right) \delta 7.80(\mathrm{dd}, J=5.8,2.3 \mathrm{~Hz}, 1 \mathrm{H})$, $7.46-7.39(\mathrm{~m}, 2 \mathrm{H}), 7.36(\mathrm{~d}, J=7.7 \mathrm{~Hz}, 1 \mathrm{H}), 7.25-7.22(\mathrm{~m}$, $3 \mathrm{H}), 7.22-7.13(\mathrm{~m}, 2 \mathrm{H}), 5.14(\mathrm{~d}, J=14.5 \mathrm{~Hz}, 1 \mathrm{H}), 4.87(\mathrm{~s}$, $1 \mathrm{H}), 4.24(\mathrm{~d}, J=14.5 \mathrm{~Hz}, 1 \mathrm{H}), 3.56(\mathrm{t}, J=4.5 \mathrm{~Hz}, 4 \mathrm{H})$, 2.63-2.49 (m, 2H), 2.43-2.29 (m, 2H); ${ }^{13} \mathrm{C}$ NMR (100 MHz, $\left.\mathrm{CDCl}_{3} / \mathrm{CCl}_{4}, 1 / 1\right) \delta 167.2(\mathrm{C}), 141.7(\mathrm{C}), 137.3(\mathrm{C}), 132.8$ $(\mathrm{CH}), 131.3(\mathrm{C}), 129.1(\mathrm{CH}), 128.6(\mathrm{CH}), 128.4(\mathrm{CH}), 127.4$ $(\mathrm{CH}), 123.9(\mathrm{CH}), 123.4(\mathrm{CH}), 77.2(\mathrm{CH}), 67.2\left(\mathrm{CH}_{2}\right), 47.6$ $\left(\mathrm{CH}_{2}\right), 43.5\left(\mathrm{CH}_{2}\right)$; HRMS (ESI) calcd. for $\mathrm{C}_{19} \mathrm{H}_{21} \mathrm{~N}_{2} \mathrm{O}_{2}(\mathrm{M}$ + H) 309.1603, found 309.1594 .

\section{2i 2-Benzyl-3-(S)-1-phenylethyl)amino)isoindolin-} 1-one (7i):

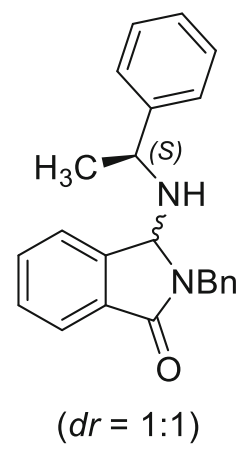

Yellow liquid (95 mg, 66\% yield); IR $\left(\mathrm{KBr}, \mathrm{cm}^{-1}\right) 3493$, 3335, 3032, 2968, 2923, 1764, 1688, 1460, 1409, 1360, 1276, $1212,1081,975,747,700 ;{ }^{1} \mathrm{H}$ NMR $\left(400 \mathrm{MHz}, \mathrm{CDCl}_{3}\right) \delta$ 7.82-7.75 (m, 2H), 7.51-7.47 (m, 1H), 7.46-7.40 (m, 2H), 7.34-7.30 (m, 2H), $7.28(\mathrm{t}, J=4.1 \mathrm{~Hz}, 2 \mathrm{H}), 7.24(\mathrm{~s}, 1 \mathrm{H})$, $7.22(\mathrm{~d}, J=1.8 \mathrm{~Hz}, 2 \mathrm{H}), 7.21-7.20(\mathrm{~m}, 2 \mathrm{H}), 7.19(\mathrm{~d}, J=$ $1.4 \mathrm{~Hz}, 2 \mathrm{H}), 7.18(\mathrm{~s}, 3 \mathrm{H}), 7.16(\mathrm{~d}, J=2.8 \mathrm{~Hz}, 2 \mathrm{H}), 7.14(\mathrm{t}$, $J=2.7 \mathrm{~Hz}, 2 \mathrm{H}), 7.13-7.11(\mathrm{~m}, 5 \mathrm{H}), 5.16(\mathrm{~d}, J=14.9 \mathrm{~Hz}$, $1 \mathrm{H}), 5.09(\mathrm{~s}, 1 \mathrm{H}), 5.05(\mathrm{~s}, 1 \mathrm{H}), 4.91(\mathrm{~d}, J=15.3 \mathrm{~Hz}, 1 \mathrm{H})$, $4.34(\mathrm{~d}, J=15.0 \mathrm{~Hz}, 1 \mathrm{H}), 3.98(\mathrm{~d}, J=15.4 \mathrm{~Hz}, 1 \mathrm{H}), 3.83$ $(\mathrm{q}, J=6.6 \mathrm{~Hz}, 1 \mathrm{H}), 3.70(\mathrm{t}, J=6.6 \mathrm{~Hz}, 1 \mathrm{H}), 2.08(\mathrm{~s}, 2 \mathrm{H})$, $1.24(\mathrm{~d}, J=6.6 \mathrm{~Hz}, 3 \mathrm{H}), 1.18(\mathrm{~d}, J=6.7 \mathrm{~Hz}, 3 \mathrm{H}) ;{ }^{13} \mathrm{C}$ NMR (100 MHz, $\left.\mathrm{CDCl}_{3}\right) \delta 167.6(\mathrm{C}), 167.4(\mathrm{C}), 145.7$ (C), 145.4 (C), 144.49 (C), 144.40 (C), 137.56 (C), 137.53 (C), $132.3(\mathrm{C}), 131.9(\mathrm{C}), 131.7(\mathrm{CH}), 131.2(\mathrm{CH}), 129.0(\mathrm{CH})$, $128.7(\mathrm{CH}), 128.67(\mathrm{CH}), 128.62(\mathrm{CH}), 128.60(\mathrm{CH}), 128.3$ $(\mathrm{CH}), 128.2(\mathrm{CH}), 128.1(\mathrm{CH}), 127.8(\mathrm{CH}), 127.35(\mathrm{CH})$, $127.30(\mathrm{CH}), 126.9(\mathrm{CH}), 126.7(\mathrm{CH}), 126.4(\mathrm{CH}), 123.4$ $(\mathrm{CH}), 123.3(\mathrm{CH}), 123.28(\mathrm{CH}), 123.24(\mathrm{CH}), 71.5(\mathrm{CH})$, $71.0(\mathrm{CH}), 53.8(\mathrm{CH}), 52.8(\mathrm{CH}), 42.9\left(\mathrm{CH}_{2}\right), 42.8\left(\mathrm{CH}_{2}\right)$,
$25.0(\mathrm{CH}), 24.7(\mathrm{CH})$; HRMS (ESI) calcd. for $\mathrm{C}_{23} \mathrm{H}_{23} \mathrm{~N}_{2} \mathrm{O}$ $(\mathrm{M}+\mathrm{H})$ 343.1810, found 343.1817.

\section{$2.2 \mathrm{j} \quad$ 2-Benzyl-3-(R)-1-phenylethyl)amino)isoindolin- 1-one $(\mathbf{7 j})$ :}<smiles>C[C@H](N[C@H]1c2ccccc2C(=O)N1Cc1ccccc1)c1ccccc1</smiles>

Yellow liquid (90 mg, 62\% yield); IR $\left(\mathrm{KBr}, \mathrm{cm}^{-1}\right) 3452$, $3335,3028,2970,2924,1758,1690,1459,1407,1359,1280$, $1211,1078,839,746,700 ;{ }^{1} \mathrm{HNMR}\left(400 \mathrm{MHz}, \mathrm{CDCl}_{3} / \mathrm{CCl}_{4}\right.$, 1/1) $\delta 7.75(\mathrm{~d}, J=7.5 \mathrm{~Hz}, 1 \mathrm{H}), 7.70(\mathrm{~d}, J=7.5 \mathrm{~Hz}, 1 \mathrm{H})$, $7.43(\mathrm{~d}, J=7.3 \mathrm{~Hz}, 1 \mathrm{H}), 7.41-7.39(\mathrm{~m}, 1 \mathrm{H}), 7.37(\mathrm{~d}, J=$ $6.7 \mathrm{~Hz}, 1 \mathrm{H}), 7.27(\mathrm{~s}, 1 \mathrm{H}), 7.24(\mathrm{~s}, 1 \mathrm{H}), 7.22(\mathrm{~s}, 2 \mathrm{H}), 7.19$ $(\mathrm{d}, J=5.4 \mathrm{~Hz}, 3 \mathrm{H}), 7.17(\mathrm{~d}, J=3.4 \mathrm{~Hz}, 3 \mathrm{H}), 7.14(\mathrm{~d}$, $J=4.7 \mathrm{~Hz}, 2 \mathrm{H}), 7.11(\mathrm{~s}, 3 \mathrm{H}), 7.10(\mathrm{~s}, 2 \mathrm{H}), 7.08(\mathrm{~s}, 2 \mathrm{H})$, 7.06 (s, 2H), 7.05 (s, 2H), , 5.10 (d, $J=14.9 \mathrm{~Hz}, 1 \mathrm{H}), 5.02$ (s, 1H), 4.99 (s, 1H), 4.85 (d, $J=15.3 \mathrm{~Hz}, 1 \mathrm{H}), 4.27$ (d, $J=14.9 \mathrm{~Hz}, 1 \mathrm{H}), 3.89(\mathrm{~d}, J=15.3 \mathrm{~Hz}, 1 \mathrm{H}), 3.78(\mathrm{q}, J=$ $6.6 \mathrm{~Hz}, 1 \mathrm{H}), 3.63(\mathrm{q}, J=6.6 \mathrm{~Hz}, 1 \mathrm{H}), 1.89(\mathrm{~s}, 2 \mathrm{H}), 1.20(\mathrm{~d}$, $J=6.6 \mathrm{~Hz}, 3 \mathrm{H}), 1.12(\mathrm{~d}, J=6.6 \mathrm{~Hz}, 3 \mathrm{H}) ;{ }^{13} \mathrm{C}$ NMR $(100$ $\left.\mathrm{MHz}, \mathrm{CDCl}_{3} / \mathrm{CCl}_{4}, 1 / 1\right) \delta 167.5$ (C), 167.3 (C), 145.9 (C), 145.5 (C), 144.6 (C), 144.5 (C), 137.78 (C), 137.71(C), 132.5 (C), $132.2(\mathrm{C}), 131.7(\mathrm{CH}), 131.2(\mathrm{CH}), 130.0(\mathrm{CH}), 129.1$ $(\mathrm{CH}), 128.82(\mathrm{CH}), 128.80(\mathrm{CH}), 128.7(\mathrm{CH}), 128.4(\mathrm{CH})$, $128.3(\mathrm{CH}), 127.9(\mathrm{CH}), 127.5(\mathrm{CH}), 127.1(\mathrm{CH}), 126.8(\mathrm{CH})$, $126.5(\mathrm{CH}), 126.0(\mathrm{CH}) 123.58(\mathrm{CH}), 123.56(\mathrm{CH}), 123.54$ $(\mathrm{CH}), 123.3(\mathrm{CH}), 122.1(\mathrm{CH}), 71.6(\mathrm{CH}), 71.0(\mathrm{CH}), 54.0$ $(\mathrm{CH}), 53.0(\mathrm{CH}), 43.2\left(\mathrm{CH}_{2}\right), 43.0\left(\mathrm{CH}_{2}\right), 25.3(\mathrm{CH}), 24.9$ (CH); HRMS (ESI) calcd. for $\mathrm{C}_{23} \mathrm{H}_{23} \mathrm{~N}_{2} \mathrm{O}(\mathrm{M}+\mathrm{H}) 343.1810$, found 343.1801 .

\section{2k 3-(Dimethylamino)-2-(4-methoxybenzyl)} isoindolin-1-one (7k):<smiles>CN(C)C1c2ccccc2C(=O)N1C(C)(C)C</smiles>

White solid (102 mg, $92 \%$ yield); M.p. $115^{\circ} \mathrm{C}$; IR (KBr, $\left.\mathrm{cm}^{-1}\right) 3480,2939,2836,2791,1763,1691,1614,1512,1464$, 1411, 1361, 1248, 1180, 1100, 1038, 836, 746, 692, 595, 534; ${ }^{1} \mathrm{H}$ NMR $\left(400 \mathrm{MHz}, \mathrm{CDCl}_{3} / \mathrm{CCl}_{4}, 1 / 1\right) \delta 7.82(\mathrm{dd}, J=5.8$, $2.7 \mathrm{~Hz}, 1 \mathrm{H}), 7.47-7.40(\mathrm{~m}, 2 \mathrm{H}), 7.38(\mathrm{~d}, J=1.9 \mathrm{~Hz}, 1 \mathrm{H})$, $7.21(\mathrm{~d}, J=8.6 \mathrm{~Hz}, 2 \mathrm{H}), 6.78(\mathrm{~d}, J=8.7 \mathrm{~Hz}, 2 \mathrm{H}), 5.15(\mathrm{~d}$, $J=14.5 \mathrm{~Hz}, 1 \mathrm{H}), 4.95(\mathrm{~s}, 1 \mathrm{H}), 4.13(\mathrm{~d}, J=14.5 \mathrm{~Hz}, 1 \mathrm{H})$, 
Table 1. Crystallographic data and structure refinement for $7 \mathbf{k}$.

\begin{tabular}{lc}
\hline Empirical Formula & $\mathrm{C}_{18} \mathrm{H}_{20} \mathrm{~N}_{2} \mathrm{O}_{2}$ \\
Formula Weight & 296.37 \\
Bond precision & $\mathrm{C}-\mathrm{C} 0.0026 \AA$ \\
Wavelength & 0.71073 \\
a, $\AA$ & $11.8224(9)$ \\
$\mathrm{b}, \AA$ & $6.2257(5)$ \\
$\mathrm{c}, \AA$ & $21.7641(17)$ \\
$\alpha,{ }^{\circ}$ & 90 \\
$\beta, \circ$ & $90.456(7)$ \\
$\gamma, \circ$ & 90 \\
Temperature & $295 \mathrm{~K}$ \\
Volume & $1601.9(2)$ \\
Space group & $\mathrm{P} 21 / \mathrm{c}$ \\
Hall group & $-\mathrm{P} 2 \mathrm{ybc}$ \\
Mr & 296.36 \\
Dx, g cm-3 & 1.229 \\
$\mathrm{Z}$ & 4 \\
Mu (mm-1) & 0.081 \\
F000 & 632.0 \\
F000' & 632.26 \\
h,k,lmax & $16,8,29$ \\
Nref & 4287 \\
Tmin,Tmax & $0.975,0.992$ \\
Tmin' & 0.941 \\
Correction method: & \\
TminTmax & $0.707,0.922$ \\
Data completeness & 0.774 \\
Theta(max) & 29.060 \\
R(reflections) & $0.0466(2295)$ \\
wR2(reflections) & $0.1332(3320)$ \\
S & 1.086 \\
Npar & 201 \\
\hline
\end{tabular}

3.73 (s, 3H), 2.25 (s, 6H); ${ }^{13} \mathrm{C} \mathrm{NMR}\left(100 \mathrm{MHz}, \mathrm{CDCl}_{3} / \mathrm{CCl}_{4}\right.$, 1/1) $\delta 167.1(\mathrm{C}), 158.9(\mathrm{C}), 142.2(\mathrm{C}), 132.9(\mathrm{C}), 131.1(\mathrm{CH})$, $129.7(\mathrm{CH}), 129.5(\mathrm{C}), 128.9(\mathrm{CH}), 123.7(\mathrm{CH}), 123.3(\mathrm{CH})$, $113.9(\mathrm{CH}), 77.1(\mathrm{CH}), 55.0(\mathrm{CH}), 42.7\left(\mathrm{CH}_{2}\right), 38.9(\mathrm{CH})$; HRMS (ESI) calcd. for $\mathrm{C}_{18} \mathrm{H}_{21} \mathrm{~N}_{2} \mathrm{O}_{2}(\mathrm{M}+\mathrm{H})$ 297.1603, found 297.1600 (Table 1).

2.21 2-(4-Methoxybenzyl)-3-(phenylamino)isoindolin1-one (7l):<smiles>CC(C)(C)N1C(=O)c2ccccc2C1Nc1ccccc1</smiles>

White solid (108 mg, 85\% yield); M.p. $150^{\circ} \mathrm{C}$; IR ( $\mathrm{KBr}$, $\mathrm{cm}^{-1}$ ); 3315, 2924, 2839, 1689, 1604, 1512, 1407, 1301, $1249,1173,1098,1030,972,902,824,749,694,546 ;{ }^{1} \mathrm{H}$ NMR $\left(400 \mathrm{MHz}, \mathrm{CDCl}_{3} / \mathrm{CCl}_{4}, 1 / 1\right) \delta 7.81-7.76(\mathrm{~m}, 1 \mathrm{H})$,
7.50-7.44 (m, 2H), $7.43(\mathrm{~d}, J=2.0 \mathrm{~Hz}, 1 \mathrm{H}), 7.14(\mathrm{dd}$, $J=8.5,7.4 \mathrm{~Hz}, 2 \mathrm{H}), 7.08(\mathrm{~d}, J=8.6 \mathrm{~Hz}, 2 \mathrm{H}), 6.84-6.78$ $(\mathrm{m}, 1 \mathrm{H}), 6.78-6.75(\mathrm{~m}, 2 \mathrm{H}), 6.61(\mathrm{~d}, J=1.0 \mathrm{~Hz}, 1 \mathrm{H}), 6.59$ $(\mathrm{d}, J=0.9 \mathrm{~Hz}, 1 \mathrm{H}), 5.80(\mathrm{~s}, 1 \mathrm{H}), 5.05(\mathrm{~d}, J=14.9 \mathrm{~Hz}$, $1 \mathrm{H}), 4.06(\mathrm{~d}, J=14.9 \mathrm{~Hz}, 1 \mathrm{H}), 3.76(\mathrm{~s}, 3 \mathrm{H}) ;{ }^{13} \mathrm{C} \mathrm{NMR}$ (100 MHz, $\left.\mathrm{CDCl}_{3} / \mathrm{CCl}_{4}, 1 / 1\right) \delta 167.1$ (C), 159.1 (C), 145.7 (C), $144.1(\mathrm{C}), 132.1(\mathrm{C}), 132.0(\mathrm{CH}), 129.7(\mathrm{C}), 129.6(\mathrm{CH})$, $129.49(\mathrm{CH}), 129.46(\mathrm{CH}), 123.7(\mathrm{CH}), 123.2(\mathrm{CH}), 119.5$ $(\mathrm{CH}), 114.5(\mathrm{CH}), 114.1(\mathrm{CH}), 68.64(\mathrm{CH}), 55.24(\mathrm{CH})$, $42.58\left(\mathrm{CH}_{2}\right)$; HRMS (ESI) calcd. for $\mathrm{C}_{22} \mathrm{H}_{21} \mathrm{~N}_{2} \mathrm{O}_{2}(\mathrm{M}+\mathrm{H})$ 345.1603 , found 345.1610 .

\section{$2.2 \mathrm{~m} \quad 3-($ Cyclohexylamino $)-2-(4-m e t h o x y b e n z y l)$ isoindolin-1-one (7m):}<smiles>CC(C)(C)N1C(=O)c2ccccc2C1NC1CCCCC1</smiles>

White solid (113 mg, 86\% yield); M.p. $113^{\circ} \mathrm{C}$; IR (KBr, $\mathrm{cm}^{-1}$ ) 3337, 2928, 2847, 1679, 1510, 1467, 1391, 1352, 1306, 1236, 1176, 1121, 1090, 1032, 964, 842, 746, 694, 620, 520; ${ }^{1} \mathrm{H}$ NMR $\left(400 \mathrm{MHz}, \mathrm{CDCl}_{3} / \mathrm{CCl}_{4}, 1 / 1\right) \delta 7.82$ $(\mathrm{d}, J=6.9 \mathrm{~Hz}, 1 \mathrm{H}), 7.46(\mathrm{dt}, J=8.8,3.5 \mathrm{~Hz}, 3 \mathrm{H}), 7.21$ $(\mathrm{d}, J=8.6 \mathrm{~Hz}, 2 \mathrm{H}), 6.80(\mathrm{~d}, J=8.6 \mathrm{~Hz}, 2 \mathrm{H}), 5.18(\mathrm{~d}$, $J=14.8 \mathrm{~Hz}, 1 \mathrm{H}), 5.14(\mathrm{~s}, 1 \mathrm{H}), 4.20(\mathrm{~d}, J=14.8 \mathrm{~Hz}, 1 \mathrm{H})$, $3.76(\mathrm{~s}, 3 \mathrm{H}), 2.48-2.40(\mathrm{~m}, 1 \mathrm{H}), 1.75-1.71(\mathrm{~m}, 1 \mathrm{H}), 1.70$ $1.63(\mathrm{~m}, 2 \mathrm{H}), 1.62-1.57(\mathrm{~m}, 1 \mathrm{H}), 1.54-1.45(\mathrm{~m}, 2 \mathrm{H}), 1.15(\mathrm{~d}$, $J=7.4 \mathrm{~Hz}, 2 \mathrm{H}), 1.12-1.06(\mathrm{~m}, 2 \mathrm{H}), 1.00(\mathrm{dd}, J=11.1,8.3$ $\mathrm{Hz}, 1 \mathrm{H}),{ }^{13} \mathrm{C}$ NMR $\left(100 \mathrm{MHz}, \mathrm{CDCl}_{3} / \mathrm{CCl}_{4}, 1 / 1\right) \delta 167.3(\mathrm{C})$, $159.1(\mathrm{C}), 145.2(\mathrm{C}), 132.6(\mathrm{C}), 131.6(\mathrm{CH}), 129.8(\mathrm{C}), 129.5$ $(\mathrm{CH}), 129.0(\mathrm{CH}), 123.6(\mathrm{CH}), 123.3(\mathrm{CH}), 114.2(\mathrm{CH}), 71.1$ $(\mathrm{CH}), 55.2(\mathrm{CH}), 51.8(\mathrm{CH}), 42.4\left(\mathrm{CH}_{2}\right), 35.4\left(\mathrm{CH}_{2}\right), 35.1$ $\left(\mathrm{CH}_{2}\right), 25.9\left(\mathrm{CH}_{2}\right), 25.1\left(\mathrm{CH}_{2}\right), 24.9\left(\mathrm{CH}_{2}\right)$; HRMS (ESI) calcd. for $\mathrm{C}_{22} \mathrm{H}_{27} \mathrm{~N}_{2} \mathrm{O}_{2}(\mathrm{M}+\mathrm{H})$ 351.2073, found 351.2099.

2.2n 3-(Benzylamino)-2-(4-methoxybenzyl)isoindolin1-one (7n):<smiles>CC(C)(C)N1C(=O)c2ccccc2C1NCc1ccccc1</smiles>

Colorless liquid (119 mg, 89\% yield); IR $\left(\mathrm{KBr}, \mathrm{cm}^{-1}\right) 3333$, 2915, 2841, 1690, 1611, 1510, 1458, 1401, 1356, 1245, 1176, 1086, 1033, 967, 903, 836, 746, 699, 587; ${ }^{1} \mathrm{HNMR}(400 \mathrm{MHz}$, $\left.\mathrm{CDCl}_{3} / \mathrm{CCl}_{4}, 1 / 1\right) \delta 7.86(\mathrm{~d}, J=7.3 \mathrm{~Hz}, 1 \mathrm{H}), 7.51(\mathrm{~s}, 2 \mathrm{H})$, $7.47(\mathrm{dd}, J=7.9,5.1 \mathrm{~Hz}, 1 \mathrm{H}), 7.28(\mathrm{~s}, 1 \mathrm{H}), 7.24(\mathrm{~s}, 2 \mathrm{H})$, 
$7.21(\mathrm{~d}, J=7.0 \mathrm{~Hz}, 2 \mathrm{H}), 7.15(\mathrm{~d}, J=6.9 \mathrm{~Hz}, 2 \mathrm{H}), 6.82(\mathrm{~d}$, $J=8.6 \mathrm{~Hz}, 2 \mathrm{H}), 5.31(\mathrm{~s}, 1 \mathrm{H}), 5.10(\mathrm{~d}, J=14.8 \mathrm{~Hz}, 1 \mathrm{H})$, $4.27(\mathrm{~d}, J=14.8 \mathrm{~Hz}, 1 \mathrm{H}), 3.75(\mathrm{~s}, 3 \mathrm{H}), 3.35(\mathrm{~d}, J=13.0 \mathrm{~Hz}$, $1 \mathrm{H}), 3.24(\mathrm{~d}, J=13.0 \mathrm{~Hz}, 1 \mathrm{H}), 2.14(\mathrm{~s}, 1 \mathrm{H}) ;{ }^{13} \mathrm{C} \mathrm{NMR}$ (100 MHz, $\left.\mathrm{CDCl}_{3} / \mathrm{CCl}_{4}, 1 / 1\right) \delta 167.7$ (C), 159.1 (C), 143.3 (C), $139.7(\mathrm{C}), 132.9(\mathrm{C}), 131.8(\mathrm{CH}), 129.6(\mathrm{C}), 129.5(\mathrm{CH})$, 129.2 (CH), $128.4(\mathrm{CH}), 128.1(\mathrm{CH}), 127.2(\mathrm{CH}), 123.5(\mathrm{CH})$, $123.2(\mathrm{CH}), 114.2(\mathrm{CH}), 72.2(\mathrm{CH}), 55.3(\mathrm{CH}), 45.6\left(\mathrm{CH}_{2}\right)$, $42.7\left(\mathrm{CH}_{2}\right)$; HRMS (ESI) calcd. for $\mathrm{C}_{23} \mathrm{H}_{23} \mathrm{~N}_{2} \mathrm{O}_{2}(\mathrm{M}+\mathrm{H})$ 359.1760 , found 359.1747 .

\section{$2.20 \quad 2-(4-M e t h o x y b e n z y l)-3-($ phenethylamino $)$} isoindolin-1-one (7o):

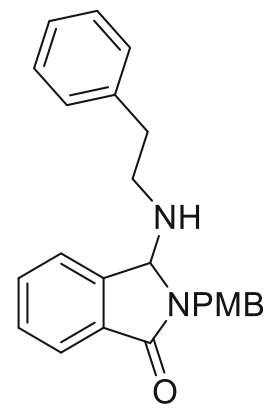

Viscous solid (116 mg, 84\% yield); IR ( $\left.\mathrm{KBr}, \mathrm{cm}^{-1}\right) 3446$, 2923, 2837, 1685, 1610, 1463, 1406, 1247, 1177, 1089, 1031, 808, 750, 696, 464; ${ }^{1} \mathrm{H}$ NMR (400 MHz, $\left.\mathrm{CDCl}_{3} / \mathrm{CCl}_{4}, 1 / 1\right)$ $\delta 7.75(\mathrm{dd}, J=6.5,1.1 \mathrm{~Hz}, 1 \mathrm{H}), 7.43-7.35(\mathrm{~m}, 2 \mathrm{H}), 7.32-$ $7.28(\mathrm{~m}, 1 \mathrm{H}), 7.23(\mathrm{dd}, J=6.1,1.3 \mathrm{~Hz}, 1 \mathrm{H}), 7.18(\mathrm{~d}, J=$ $6.5 \mathrm{~Hz}, 2 \mathrm{H}), 7.08-7.03(\mathrm{~m}, 2 \mathrm{H}), 6.91(\mathrm{~d}, J=8.6 \mathrm{~Hz}, 2 \mathrm{H})$, $6.68(\mathrm{~d}, J=8.7 \mathrm{~Hz}, 2 \mathrm{H}), 5.02(\mathrm{~s}, 1 \mathrm{H}), 4.95(\mathrm{~d}, J=14.8 \mathrm{~Hz}$, $1 \mathrm{H}), 3.70(\mathrm{~d}, J=16 \mathrm{~Hz}, 1 \mathrm{H}), 3.68(\mathrm{~s}, 3 \mathrm{H}), 2.65-2.56(\mathrm{~m}$, $1 \mathrm{H}), 2.53-2.43(\mathrm{~m}, 1 \mathrm{H}), 2.38$ (ddd, $J=11.1,8.4,5.8 \mathrm{~Hz}$, 1H), 2.27-2.20 (m, 1H), 1.72 (s, 1H); ${ }^{13} \mathrm{C} \mathrm{NMR} \mathrm{(100} \mathrm{MHz,}$ $\left.\mathrm{CDCl}_{3} / \mathrm{CCl}_{4}, 1 / 1\right) \delta 167.5$ (C), 159.2 (C), 143.3 (C), 139.7 (C), 133.1 (C), 131.7 (CH), $129.6(\mathrm{C}), 129.3(\mathrm{CH}), 129.1$ $(\mathrm{CH}), 129.0(\mathrm{CH}), 128.7(\mathrm{CH}), 126.6(\mathrm{CH}), 123.6(\mathrm{CH}), 123.2$ $(\mathrm{CH}), 114.2(\mathrm{CH}), 71.9(\mathrm{CH}), 55.3(\mathrm{CH}), 42.4\left(\mathrm{CH}_{2}\right), 41.8$ $\left(\mathrm{CH}_{2}\right), 36.2\left(\mathrm{CH}_{2}\right)$; HRMS (ESI) calcd. for $\mathrm{C}_{24} \mathrm{H}_{25} \mathrm{~N}_{2} \mathrm{O}_{2}(\mathrm{M}$ + H) 373.1916, found 373.1904.

\section{$2.2 \mathrm{p}$ 2-(4-Methoxybenzyl)-3-(piperidin-1-yl)} isoindolin-1-one (7p):<smiles>CN1C(=O)c2ccccc2C1N1CCCCC1</smiles>

Yellow solid (102 mg, $82 \%$ yield); M.p. $120^{\circ} \mathrm{C}$; IR $(\mathrm{KBr}$, $\left.\mathrm{cm}^{-1}\right)$; 3451, 2933, 2845, 1767, 1693, 1612, 1511, 1459, 1408, 1246, 1172, 1095, 1038, 1003, 835, 744, 695, 520, 449; ${ }^{1} \mathrm{H}$ NMR (400 MHz, $\left.\mathrm{CDCl}_{3} / \mathrm{CCl}_{4}, 1 / 1\right) \delta 7.84$ (dd, $J=5.8,2.6 \mathrm{~Hz}, 1 \mathrm{H}), 7.45(\mathrm{t}, J=2.8 \mathrm{~Hz}, 2 \mathrm{H}), 7.39(\mathrm{~d}$,
$J=7.9 \mathrm{~Hz}, 1 \mathrm{H}), 7.26(\mathrm{~d}, J=1.5 \mathrm{~Hz}, 1 \mathrm{H}), 7.24(\mathrm{~s}, 1 \mathrm{H})$, $6.82(\mathrm{~d}, J=2.0 \mathrm{~Hz}, 1 \mathrm{H}), 6.81(\mathrm{~d}, J=2.1 \mathrm{~Hz}, 1 \mathrm{H}), 5.15$ $(\mathrm{d}, J=14.3 \mathrm{~Hz}, 1 \mathrm{H}), 4.90(\mathrm{~s}, 1 \mathrm{H}), 4.19(\mathrm{~d}, J=14.3 \mathrm{~Hz}$, $1 \mathrm{H}), 3.77(\mathrm{~s}, 3 \mathrm{H}), 2.62-2.51(\mathrm{~m}, 2 \mathrm{H}), 2.39(\mathrm{~d}, J=5.0 \mathrm{~Hz}$, $2 \mathrm{H}), 1.54(\mathrm{~d}, J=5.0 \mathrm{~Hz}, 4 \mathrm{H}), 1.46(\mathrm{~d}, J=4.2 \mathrm{~Hz}, 2 \mathrm{H}),{ }^{13} \mathrm{C}$ NMR (100 MHz, $\left.\mathrm{CDCl}_{3} / \mathrm{CCl}_{4}, 1 / 1\right) \delta 167.2$ (C), $159.0(\mathrm{C})$, $142.9(\mathrm{C}), 133.2(\mathrm{C}), 131.1(\mathrm{CH}), 130.0(\mathrm{CH}), 129.9(\mathrm{CH})$, $128.9(\mathrm{CH}), 123.9(\mathrm{CH}), 123.5(\mathrm{CH}), 114.0(\mathrm{CH}), 77.8(\mathrm{CH})$, $55.3(\mathrm{CH}), 48.6\left(\mathrm{CH}_{2}\right), 42.8\left(\mathrm{CH}_{2}\right), 26.6\left(\mathrm{CH}_{2}\right), 25.0\left(\mathrm{CH}_{2}\right)$; HRMS (ESI) calcd. for $\mathrm{C}_{21} \mathrm{H}_{25} \mathrm{~N}_{2} \mathrm{O}_{2}(\mathrm{M}+\mathrm{H}) 337.1916$, found 337.1921.

2.2q 2-(4-Methoxybenzyl)-3-morpholinoisoindolin-1one $(\mathbf{7 q})$ :<smiles>CN1C(=O)c2ccccc2C1N1CCOCC1</smiles>

White solid (99 mg, $79 \%$ yield); M.p. $130^{\circ} \mathrm{C}$; IR ( $\mathrm{KBr}$, $\mathrm{cm}^{-1}$ ); 3240, 2923, 2703, 1670, 1440, 1307, 1211, 1118, $1058,960,919,791,742,698,633,600,520 ;{ }^{1} \mathrm{H}$ NMR (400 $\left.\mathrm{MHz}, \mathrm{CDCl}_{3} / \mathrm{CCl}_{4}, 1 / 1\right) \delta 7.88-7.83(\mathrm{~m}, 1 \mathrm{H}), 7.51-7.45(\mathrm{~m}$, $2 \mathrm{H}), 7.42(\mathrm{dd}, J=5.9,2.5 \mathrm{~Hz}, 1 \mathrm{H}), 7.26(\mathrm{~s}, 1 \mathrm{H}), 7.24(\mathrm{~d}$, $J=1.9 \mathrm{~Hz}, 1 \mathrm{H}), 6.84-6.82(\mathrm{~m}, 1 \mathrm{H}), 6.82-6.79(\mathrm{~m}, 1 \mathrm{H}), 5.17$ $(\mathrm{d}, J=14.4 \mathrm{~Hz}, 1 \mathrm{H}), 4.92(\mathrm{~s}, 1 \mathrm{H}), 4.23(\mathrm{~d}, J=14.4 \mathrm{~Hz}$, $1 \mathrm{H}), 3.78(\mathrm{~s}, 3 \mathrm{H}), 3.66(\mathrm{t}, J=4.6 \mathrm{~Hz}, 4 \mathrm{H}), 2.71-2.60(\mathrm{~m}$, 2H), 2.49-2.41 (m, 2H); ${ }^{13} \mathrm{C}$ NMR (100 MHz, $\mathrm{CDCl}_{3} / \mathrm{CCl}_{4}$, 1/1) $\delta 167.3$ (C), 159.2 (C), 142.0 (C), 133.1 (C), 131.4 (C), $130.0(\mathrm{CH}), 129.6(\mathrm{CH}), 129.3(\mathrm{CH}), 124.1(\mathrm{CH}), 123.5(\mathrm{CH})$, 114.1 $(\mathrm{CH}), 77.3(\mathrm{CH}), 67.4\left(\mathrm{CH}_{2}\right), 55.3(\mathrm{CH}), 47.8\left(\mathrm{CH}_{2}\right)$, $43.1\left(\mathrm{CH}_{2}\right)$ HRMS (ESI) calcd. for $\mathrm{C}_{20} \mathrm{H}_{23} \mathrm{~N}_{2} \mathrm{O}_{3}(\mathrm{M}+\mathrm{H})$ 339.1709 , found 339.1709 .

2.2r 2-(4-Methoxybenzyl)-3-(4-phenylpiperazin-1-yl) isoindolin-1-one (7r):<smiles>CC(C)(C)N1C(=O)c2ccccc2C1N1CCN(c2ccccc2)CC1</smiles>

Viscous solid (120 mg, 78\% yield); IR $\left(\mathrm{KBr}, \mathrm{cm}^{-1}\right) 2933$, 2833, 1701, 1603, 1507, 1452, 1394, 1344, 1302, 1244, 1152, 1095, 1018, 967, 836, 752, 527, 479; ${ }^{1} \mathrm{H}$ NMR $(400 \mathrm{MHz}$, $\left.\mathrm{CDCl}_{3} / \mathrm{CCl}_{4}, 1 / 1\right) \delta 7.89-7.87(\mathrm{~m}, 1 \mathrm{H}), 7.50-7.47(\mathrm{~m}, 2 \mathrm{H})$, $7.44(\mathrm{dd}, J=5.8,2.8 \mathrm{~Hz}, 1 \mathrm{H}), 7.37(\mathrm{~d}, J=8.6 \mathrm{~Hz}, 1 \mathrm{H})$, 
$7.27(\mathrm{~s}, 1 \mathrm{H}), 7.22(\mathrm{~d}, J=7.4 \mathrm{~Hz}, 2 \mathrm{H}), 6.88(\mathrm{~d}, J=7.8 \mathrm{~Hz}$, $2 \mathrm{H}), 6.84-6.81(\mathrm{~m}, 3 \mathrm{H}), 5.21(\mathrm{~d}, J=14.4 \mathrm{~Hz}, 1 \mathrm{H}), 5.02(\mathrm{~s}$, $1 \mathrm{H}), 4.24(\mathrm{~d}, J=14.4 \mathrm{~Hz}, 1 \mathrm{H}), 3.78(\mathrm{~s}, 3 \mathrm{H}), 3.15(\mathrm{t}, J=4.8$ $\mathrm{Hz}, 4 \mathrm{H}), 2.80$ (dd, $J=10.4,5.1 \mathrm{~Hz}, 2 \mathrm{H}), 2.64-2.60(\mathrm{~m}, 2 \mathrm{H})$; ${ }^{13} \mathrm{C} \mathrm{NMR}\left(100 \mathrm{MHz}, \mathrm{CDCl}_{3} / \mathrm{CCl}_{4}, 1 / 1\right) \delta 167.3$ (C), 159.2 (C), $151.5(\mathrm{C}), 142.2(\mathrm{C}), 133.9(\mathrm{C}), 133.1(\mathrm{CH}), 131.4(\mathrm{CH})$, $130.0(\mathrm{CH}), 129.7(\mathrm{C}), 129.3(\mathrm{CH}), 124.1(\mathrm{CH}), 123.6(\mathrm{CH})$, $120.3(\mathrm{CH}), 116.6(\mathrm{CH}), 114.2(\mathrm{CH}), 77.0(\mathrm{CH}) 55.3(\mathrm{CH})$, $50.1\left(\mathrm{CH}_{2}\right), 47.4\left(\mathrm{CH}_{2}\right), 41.2\left(\mathrm{CH}_{2}\right)$; HRMS (ESI) calcd. for $\mathrm{C}_{26} \mathrm{H}_{28} \mathrm{~N}_{3} \mathrm{O}_{2}(\mathrm{M}+\mathrm{H})$ 414.2182, found 414.2213.

2.2s 3-(Dimethylamino)-2-(2-nitrobenzyl)isoindolin1-one (7s):<smiles>CN(C)C1c2ccccc2C(=O)N1C(C)(C)C</smiles>

White solid (59 mg, 54\% yield); M.p. $105^{\circ} \mathrm{C}$; IR ( $\mathrm{KBr}$, $\mathrm{cm}^{-1}$ ) 2937, 2867, 2784, 1701, 1611, 1528, 1461, 1410, 1352, 1290, 1036, 979, 856, 791, 743, 568; ${ }^{1} \mathrm{H}$ NMR (400 MHz, $\left.\mathrm{CDCl}_{3} / \mathrm{CCl}_{4}, 1 / 1\right) \delta 7.99-7.95(\mathrm{~m}, 1 \mathrm{H}), 7.87(\mathrm{dd}, J=6.3$, $1.5 \mathrm{~Hz}, 1 \mathrm{H}), 7.56-7.52(\mathrm{~m}, 1 \mathrm{H}), 7.51(\mathrm{dd}, J=2.9,1.8$ $\mathrm{Hz}, 2 \mathrm{H}), 7.50-7.46$ (m, 1H), 7.46-7.42 (m, 1H), 7.40 (dd, $J=8.3,2.2 \mathrm{~Hz}, 1 \mathrm{H}), 5.26(\mathrm{~d}, J=16.4 \mathrm{~Hz}, 1 \mathrm{H}), 5.10(\mathrm{~s}$, $1 \mathrm{H}), 4.95(\mathrm{~d}, J=16.4 \mathrm{~Hz}, 1 \mathrm{H}), 2.16(\mathrm{~s}, 6 \mathrm{H}),{ }^{13} \mathrm{C}$ NMR $(100$ $\left.\mathrm{MHz}, \mathrm{CDCl}_{3} / \mathrm{CCl}_{4}, 1 / 1\right) \delta 167.8(\mathrm{C}), 148.9(\mathrm{C}), 142.1$ (C), $133.23(\mathrm{C}), 133.21(\mathrm{CH}), 132.3(\mathrm{C}), 131.5(\mathrm{CH}), 130.3(\mathrm{CH})$, $129.1(\mathrm{CH}), 127.9(\mathrm{CH}), 124.6(\mathrm{CH}), 123.9(\mathrm{CH}), 123.3(\mathrm{CH})$, $78.9(\mathrm{CH}), 40.2\left(\mathrm{CH}_{2}\right), 38.7(\mathrm{CH})$; HRMS (ESI) calcd. for $\mathrm{C}_{17} \mathrm{H}_{18} \mathrm{~N}_{3} \mathrm{O}_{3}(\mathrm{M}+\mathrm{H}) 312.1348$, found 312.1345.

\section{2t 2-Allyl-3-(dimethylamino)isoindolin-1-one (7t):}

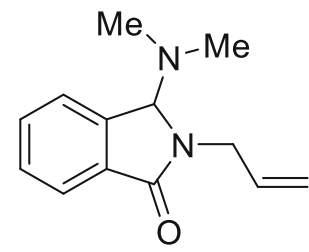

Yellow solid (83 mg, $72 \%$ yield); M.p. $55^{\circ} \mathrm{C}$; IR $\left(\mathrm{KBr}, \mathrm{cm}^{-1}\right)$ 3032, 2933, 2790, 1695, 1610, 1463, 1409, 1363, 1283, 1205, 1143, 1037, 977, 794, 743, 700, 609, 526; ${ }^{1} \mathrm{H}$ NMR $(400 \mathrm{MHz}$, $\left.\mathrm{CDCl}_{3} / \mathrm{CCl}_{4}, 1 / 1\right) \delta 7.83(\mathrm{~d}, J=6.8 \mathrm{~Hz}, 1 \mathrm{H}), 7.46(\mathrm{dd}, J=$ $10.3,3.8 \mathrm{~Hz}, 3 \mathrm{H}), 5.94-5.76(\mathrm{~m}, 1 \mathrm{H}), 5.20(\mathrm{t}, J=11.2 \mathrm{~Hz}$, $3 \mathrm{H}), 4.57$ (dd, $J=15.3,4.4 \mathrm{~Hz}, 1 \mathrm{H}), 3.77$ (dd, $J=15.3,7.4$ $\mathrm{Hz}, 1 \mathrm{H}), 2.31(\mathrm{~s}, 6 \mathrm{H}) ;{ }^{13} \mathrm{C} \mathrm{NMR}\left(100 \mathrm{MHz}, \mathrm{CDCl}_{3} / \mathrm{CCl}_{4}\right.$, 1/1) $\delta 167.3(\mathrm{C}), 142.6(\mathrm{C}), 133.6(\mathrm{C}), 133.2(\mathrm{CH}), 131.3$ $(\mathrm{CH}), 129.1(\mathrm{CH}), 123.9(\mathrm{CH}), 123.5(\mathrm{CH}), 117.7\left(\mathrm{CH}_{2}\right)$, $77.9(\mathrm{CH}), 42.7\left(\mathrm{CH}_{2}\right), 39.2(\mathrm{CH})$; HRMS (ESI) calcd. for $\mathrm{C}_{13} \mathrm{H}_{17} \mathrm{~N}_{2} \mathrm{O}(\mathrm{M}+\mathrm{H})$ 217.1341, found 217.1329.

\section{Results and Discussion}

As a first attempt, we conducted the reaction of C(3)hydroxy isoindolinone 5a with phenylboronic acid under $\mathrm{Cu}(\mathrm{OTf})_{2}$ catalysis in DMF medium under the optimized conditions worked out for the conversion of $5 \mathbf{a}$ to $6 \mathbf{a}$ (Previous work, Scheme 1). The reaction, however, provided $\mathrm{C}-\mathrm{N}$ coupled product $7 \mathbf{a}$ exclusively in $56 \%$ yield (Present work, Scheme 1). Structure of 7a was confirmed on the basis of spectroscopic (IR, ${ }^{1} \mathrm{H}$ NMR, ${ }^{13} \mathrm{C}$ NMR, DEPT-135, HSQC and HMBC 2D NMR) and analytical (ESIMS HRMS) data. Particularly, the ${ }^{1} \mathrm{H}$ NMR spectrum of 7a displayed the characteristic singlet for $\mathrm{C}(3) \mathrm{H}$ at $\delta 4.98 \mathrm{ppm}$, which was located between two doublets for diastereomeric benzylic hydrogens $(\delta 5.21$ and $4.21 \mathrm{ppm})$. As anticipated from the structure, the signal for $\mathrm{C}(3) \mathrm{H}$ of $\mathbf{7 a}$ moved to up-field by about $\delta$ $0.54 \mathrm{ppm}$ when compared to its location $(\delta 5.52 \mathrm{ppm})$ in the parent isoindolinone 5a. ${ }^{16}$ A signal for $\mathrm{C}(3)$ located at $\delta 77.3 \mathrm{ppm}$ (CH type in DEPT-135) and HMBC 2D NMR correlation between $\mathrm{C}(3) \mathrm{H}$ and NMe supported the assigned structure.

Next, we have taken up optimization of reaction conditions to increase the yield of $\mathbf{7 a}$. We conducted a series of reactions by changing the $\mathrm{Cu}$ catalysts. We tried $\mathrm{Cu}(\mathrm{II})$ catalysts like $\mathrm{Cu}\left(\mathrm{OOCCF}_{3}\right)_{2}(27 \%)$, $\mathrm{Cu}(\mathrm{OAc})_{2} \cdot \mathrm{H}_{2} \mathrm{O}(47 \%)$ and $\mathrm{CuBr}_{2}(41 \%)$ followed by $\mathrm{Cu}(\mathrm{I})$ catalysts like $\mathrm{CuI}$ (Scheme 2 and Figure 2, 80\%), $\mathrm{CuBr}(64 \%), \mathrm{CuCl}(61 \%)$ and copper(I) thiophene-2carboxylate $(\mathrm{CuTc} ; 43 \%)$. These set of experiments clearly showed that $\mathrm{CuI}$ is the best catalyst for the transformation. We found that the reaction works in open air and does not proceed under a blanket of dry $\mathrm{N}_{2}$ indicating oxygen is involved in the regeneration of the catalyst. Alternate oxidizing agents like $t$-butyl hydroperoxide (TBHP, $12 \%$ ) or $30 \%$ hydrogen peroxide $(8 \%)$ did not promote the reaction which showed their lower efficiency. By varying mol\% of $\mathrm{CuI}$ from 0.1 mol\% to 1 equivalent, we found that the transformation is best achieved with $10 \mathrm{~mol} \%$ of $\mathrm{CuI}$. The reaction did not take place in the presence of radical initiators like azobisisobutyronitrile (AIBN) or stable radical like (2,2,6,6-tetramethylpiperidin-1-yl)oxyl (TEMPO) which indicate that radical chain reactions may not be driving the reaction. Reaction worked less efficiently in presence of one equivalent of $4-\mathrm{ClC}_{6} \mathrm{H}_{4} \mathrm{~B}(\mathrm{OH})_{2}(50 \%)$ or $4-\mathrm{MeC}_{6} \mathrm{H}_{4} \mathrm{~B}(\mathrm{OH})_{2}(48 \%)$ compared to $\mathrm{C}_{6} \mathrm{H}_{5} \mathrm{~B}(\mathrm{OH})_{2}$ $(80 \%)$. Unlike the transformation of isoindolinol 5 to C-C coupled product 6 (Scheme 1, Equation 1) which required $\mathrm{Na}_{2} \mathrm{CO}_{3}$, the reaction of $\mathbf{5}$ with DMF did not require any base.

To be a successful synthetic scheme for the development of medicinal drugs, products should be derived 

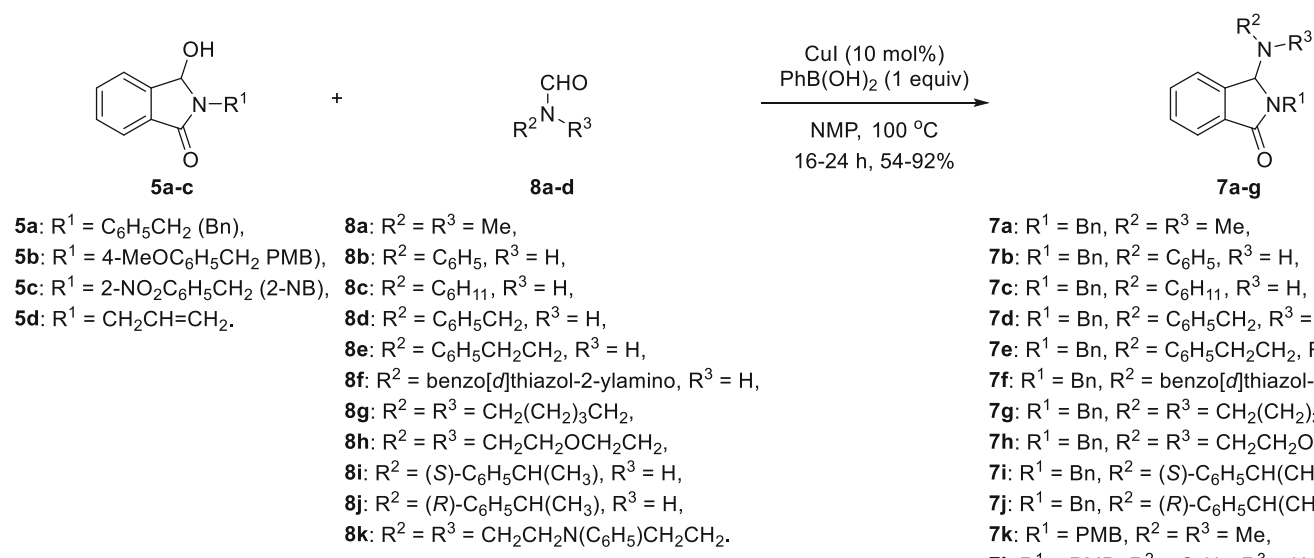

7a: $R^{1}=B n, R^{2}=R^{3}=M e$,

7b: $\mathrm{R}^{1}=\mathrm{Bn}, \mathrm{R}^{2}=\mathrm{C}_{6} \mathrm{H}_{5}, \mathrm{R}^{3}=\mathrm{H}$,

7c: $\mathrm{R}^{1}=\mathrm{Bn}, \mathrm{R}^{2}=\mathrm{C}_{6} \mathrm{H}_{11}, \mathrm{R}^{3}=\mathrm{H}$,

7d: $\mathrm{R}^{1}=\mathrm{Bn}, \mathrm{R}^{2}=\mathrm{C}_{6} \mathrm{H}_{5} \mathrm{CH}_{2}, \mathrm{R}^{3}=\mathrm{H}$,

7e: $\mathrm{R}^{1}=\mathrm{Bn}, \mathrm{R}^{2}=\mathrm{C}_{6} \mathrm{H}_{5} \mathrm{CH}_{2} \mathrm{CH}_{2}, \mathrm{R}^{3}=\mathrm{H}$,

7f: $\mathrm{R}^{1}=\mathrm{Bn}, \mathrm{R}^{2}=$ benzo[d]thiazol-2-ylamino, $\mathrm{R}^{3}=\mathrm{H}$,

7g: $\mathrm{R}^{1}=\mathrm{Bn}, \mathrm{R}^{2}=\mathrm{R}^{3}=\mathrm{CH}_{2}\left(\mathrm{CH}_{2}\right)_{3} \mathrm{CH}_{2}$,

7h: $\mathrm{R}^{1}=\mathrm{Bn}, \mathrm{R}^{2}=\mathrm{R}^{3}=\mathrm{CH}_{2} \mathrm{CH}_{2} \mathrm{OCH}_{2} \mathrm{CH}_{2}$,

7i: $\mathrm{R}^{1}=\mathrm{Bn}, \mathrm{R}^{2}=(S)-\mathrm{C}_{6} \mathrm{H}_{5} \mathrm{CH}\left(\mathrm{CH}_{3}\right), \mathrm{R}^{3}=\mathrm{H}$,

$7 \mathrm{j}: \mathrm{R}^{1}=\mathrm{Bn}, \mathrm{R}^{2}=(R)-\mathrm{C}_{6} \mathrm{H}_{5} \mathrm{CH}\left(\mathrm{CH}_{3}\right), \mathrm{R}^{3}=\mathrm{H}$,

$7 k: R^{1}=P M B, R^{2}=R^{3}=M e$,

7I: $\mathrm{R}^{1}=\mathrm{PMB}, \mathrm{R}^{2}=\mathrm{C}_{6} \mathrm{H}_{5}, \mathrm{R}^{3}=\mathrm{H}$,

$7 \mathbf{m}: \mathrm{R}^{1}=\mathrm{PMB}, \mathrm{R}^{2}=\mathrm{C}_{6} \mathrm{H}_{11}, \mathrm{R}^{3}=\mathrm{H}$,

$7 n: R^{1}=P M B, R^{2}=C_{6} H_{5} C_{2}, R^{3}=H$,

7o: $\mathrm{R}^{1}=\mathrm{PMB}, \mathrm{R}^{2}=\mathrm{C}_{6} \mathrm{H}_{5} \mathrm{CH}_{2} \mathrm{CH}_{2}, \mathrm{R}^{3}=\mathrm{H}$,

7p: $R^{1}=P M B, R^{2}=R^{3}=\mathrm{CH}_{2}\left(\mathrm{CH}_{2}\right)_{3} \mathrm{CH}_{2}$,

7q: $R^{1}=P M B, R^{2}=R^{3}=\mathrm{CH}_{2} \mathrm{CH}_{2} \mathrm{OCH}_{2} \mathrm{CH}_{2}$,

7r: $R^{1}=P M B, R^{2}=R^{3}=4$-phenylpiperazin-1-yl,

7s: $\mathrm{R}^{1}=2-\mathrm{NB}, \mathrm{R}^{2}=\mathrm{R}^{3}=\mathrm{Me}$,

7t: $\mathrm{R}^{1}=\mathrm{CH}_{2} \mathrm{CH}=\mathrm{CH}_{2}, \mathrm{R}^{2}=\mathrm{R}^{3}=\mathrm{Me}$.

Scheme 2. Copper and phenylboronic acid mediated deformylative amination of isoindolinols 5 with $N$-formyl amines 8 to form C(3)amino isoindolinins 7.

from the reactants which have readily variable domains. In the reactants of the present scheme (Scheme 2), namely isoindolinone $\mathbf{5}$ and amines $\mathbf{8}$, we envisage possibilities of changes in three domains, that is, substituents on (i) amines 8, (ii) $\mathrm{N}(2)$ and (iii) aromatic ring of $\mathbf{5}$. For the present work, we varied substituents in two domains, namely amines 8 and $\mathrm{N}(2)$ of isoindolinones 5 to generate a combinatorial library of twenty isoindolinones 7 (Scheme 2 and Figure 2). In the first instance, we employed formamides derived from primary $(\mathbf{8 b}-\mathbf{f})$ and secondary amines $(\mathbf{8 g}-\mathbf{h})$ and reacted each of them with $\mathbf{5 a}$, in $N$-methylpyrrolidone (NMP) medium in the presence of $\mathrm{CuI}$ (cat) and phenylboronic acid (stoichiometric). The reactions provided a very good yield of the isoindolinones $\mathbf{7 b - f}$. The products $\mathbf{7 e}$ and $\mathbf{7 f}$ are noteworthy as they respectively have medicinally privileged phenylethylamino and benzo $[d]$ thiozol2-ylamino moieties. Next, we employed formamides derived from secondary amines like $N$-formylpiperidine $\mathbf{8 g}$ and $N$-formylmorpholine $\mathbf{8 h}$. The reactions provided corresponding isoindolinones $\mathbf{7 g}-\mathbf{h}$ in good yield. We reasoned that the reaction with optically active $[S]$ or $[R]$ 1-phenylethylamine could provide one of the two possible diasteromers in excess. Regrettably, however, the reactions conducted with $[S] \mathbf{8 i}$ and $[R] \mathbf{8 j}$ $N$-formyl-1-phenylethylamines provided diasteromeric mixtures $\mathbf{7 i}-\mathbf{j}$ in which the diasteromers were present in $1: 1$ ratio; as evidenced by ${ }^{13} \mathrm{C}$ NMR spectra. Next, we changed benzyl group on $\mathrm{N}(2)$ to 4-methoxybenzyl $(\mathrm{PMB})$ to evaluate the influence of electron donating OMe in the outcome of the deformylative amination.
Furthermore, PMB group is amenable for removal under oxidative conditions. The series of reactions of $\mathbf{5 b}$ conducted with $N$-formylamides $\mathbf{8 a}-\mathbf{h}$ consistently provided a better yield of the products $7 \mathbf{k}-\mathbf{q}$ indicating that electron donating substituents on the benzyl ring promote substitution at $\mathrm{C}(3)$. Finally, in the series we reacted $\mathbf{5 b}$ with $\mathrm{N}(4)$ phenyl $\mathrm{N}(1)$ formylpiperizine $\mathbf{8 k}$ under optimized reaction conditions to get isoindolinone $\mathbf{7 r}$ which has many structural similarities of the developmental drug, a dopamine D4 antagonist (S)-PD172938. ${ }^{17}$ Next, we sought to evaluate if an electron-withdrawing group (e.g., $\mathrm{NO}_{2}$ ) in the $\mathrm{N}(2)$ benzyl substituent would reduce the reactivity of isoindolinone $\mathbf{5}$ with DMF. Accordingly, we reacted 5c with DMF 8a under optimized reaction conditions and realized $\mathbf{7 s}$, albeit, in lower yield. The N(2) 2nitrobenzyl group on isoindolinone $\mathbf{5 c}$ was selected because of photo-labile nature of 2-nitrobenzyl substitution. Thus, the set of reactions of DMF with 5a-c showed that electron-donating groups on $\mathrm{N}(2)$ benzyl promote deformylative amino-substitution whereas electron-withdrawing groups impede. Finally, in the present series, we conducted the reaction of isoindolinone with $N$-allyl substitution 5d with DMF and realized $7 \mathbf{t}$ without any difficulty. Structures of the isoindolinones $\mathbf{7 b - t}$ were confirmed by spectroscopic (IR, NMR) and analytical (ESIMS HRMS) data. In the case of $\mathbf{7 k}$, we recorded single crystal X-ray structure data and the out-put confirmed the assigned structure (see experimental section and supplementary information) (Figure 3). 
<smiles>CN(C)C1c2ccccc2C(=O)N1Cc1ccccc1</smiles>

$7 a, 24$ h, $80 \%$<smiles>O=C1c2ccccc2C(Nc2nc3ccccc3s2)C1Cc1ccccc1</smiles>

7f, 24 h, $80 \%$<smiles>O=C1c2ccccc2N(c2ccccc2)C1Cc1ccccc1</smiles>

7b, 24 h, $78 \%$<smiles>O=C1c2ccccc2[C@@H](N2CCCCC2)C1Cc1ccccc1</smiles>

$7 \mathrm{~g}, 21 \mathrm{~h}, 81 \%$

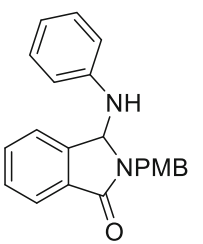

7I, 16 h, $85 \%$<smiles>O=C1c2ccccc2C(NC2CCCCC2)C1Cc1ccccc1</smiles>

7c, $22 \mathrm{~h}, 77 \%$<smiles>O=C1c2ccccc2[C@@H](N2CCOCC2)C1Cc1ccccc1</smiles>

$7 h, 23 \mathrm{~h}, 73 \%$<smiles>CS(=O)(=O)N1C(=O)c2ccccc2C1NC1CCCCC1</smiles>

$7 \mathrm{~m}, 20 \mathrm{~h}, 86 \%$<smiles>O=C1c2ccccc2C(NCc2ccccc2)N1Cc1ccccc1</smiles>

$7 d, 22$ h, $80 \%$<smiles>C[C@H](N[C@H]1C(=O)c2ccccc21)c1ccccc1</smiles>

$7 i, 24$ h, $66 \%$<smiles>CS(=O)(=O)N1C(=O)c2ccccc2C1NCc1ccccc1</smiles>

$7 n, 18$ h, $89 \%)$<smiles>O=C1c2ccccc2C(NCCc2ccccc2)N1Cc1ccccc1</smiles>

$7 e, 23 \mathrm{~h}, 75 \%$<smiles>C[C@H](N[C@H]1C(=O)c2ccccc21)c1ccccc1</smiles>

7j, 24 h, $62 \%$<smiles>CS(=O)(=O)N1C(=O)c2ccccc2C1NCCc1ccccc1</smiles>

70,17 h, $84 \%$<smiles>CCCCN1C(=O)c2ccccc2C1N1CCOCC1</smiles><smiles>CC(C)(C)N1C(=O)c2ccccc2C1N1CCN(c2ccccc2)CC1</smiles><smiles>CN(C)C1c2ccccc2C(=O)N1[15NH2]</smiles><smiles>C/C=C\CN1C(=O)c2ccccc2C1N(C)C</smiles>

$7 p, 17$ h, $82 \%$

$7 q, 20$ h, $79 \%$

$7 \mathrm{r}, 22 \mathrm{~h}, 78 \%$

$7 s, 24$ h, $54 \%$ $\mathrm{Bn}=\mathrm{C}_{6} \mathrm{H}_{5} \mathrm{CH}_{2} ; \mathrm{PMB}=4-\mathrm{MeOC}_{6} \mathrm{H}_{4} \mathrm{CH}_{2} ; 2-\mathrm{NB}=2-\mathrm{NO}_{2} \mathrm{C}_{6} \mathrm{H}_{4} \mathrm{CH}_{2}$.

Figure 2. Structures of $\mathrm{N}(2)$ and $\mathrm{C}(3)$ substituted isoindolinones 7a-t with time taken for the reaction and yield.

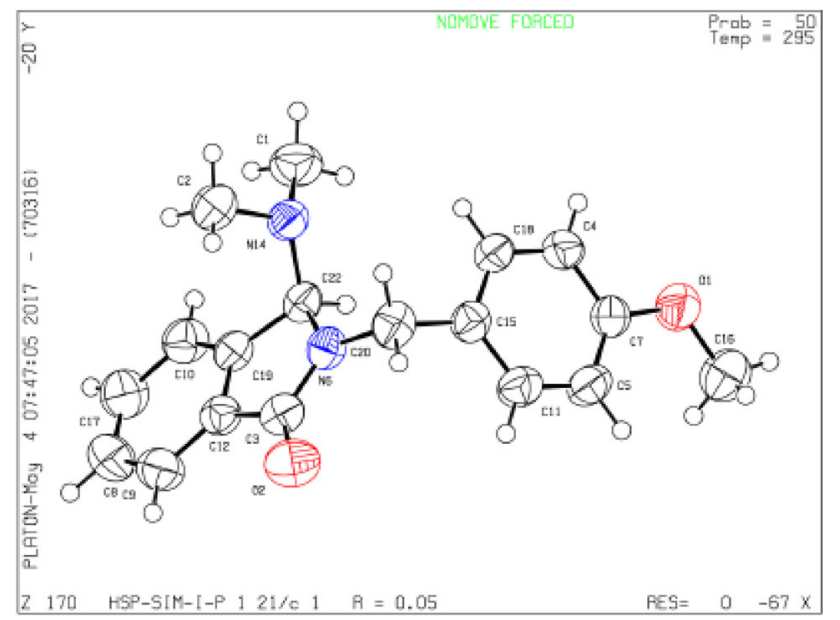

Figure 3. ORTEP diagram for 3-(dimethylamino)-2(4-methoxybenzyl)isoindolin-1-one 7k.
Based on the above results we propose a speculative mechanism as given in Scheme 3. We believe that in the first step $\mathrm{Cu}(\mathrm{I})$ reacts with phenylboronic acid to form $\mathrm{Cu}$ (III) transmetallated product 9 . Highly reactive $\mathrm{Cu}$ (III) intermediate 9 then reacts with isoindolinone 5 to form stable boric acid and organo-copper(III) intermediate 10. Reaction of $\mathbf{1 0}$ with formamides $\mathbf{8}$ leads to benzene and 11. Loss of carbon-monoxide generates 12. Final step in the sequence is the generation of $\mathrm{C}-\mathrm{N}$ coupled product 7 and $\mathrm{Cu}(\mathrm{I})$ species. The interemediate $\mathbf{1 0}$ or even 9 can generate biphenyl as the side product. Indeed, we have isolated biphenyl as a minor side product (about 20\%) in each of the reactions given in Scheme 2. In the presence of copper-chelating formamides, the reaction does not follow the path where C-C bond formation (Equation 1, Scheme 1) takes 


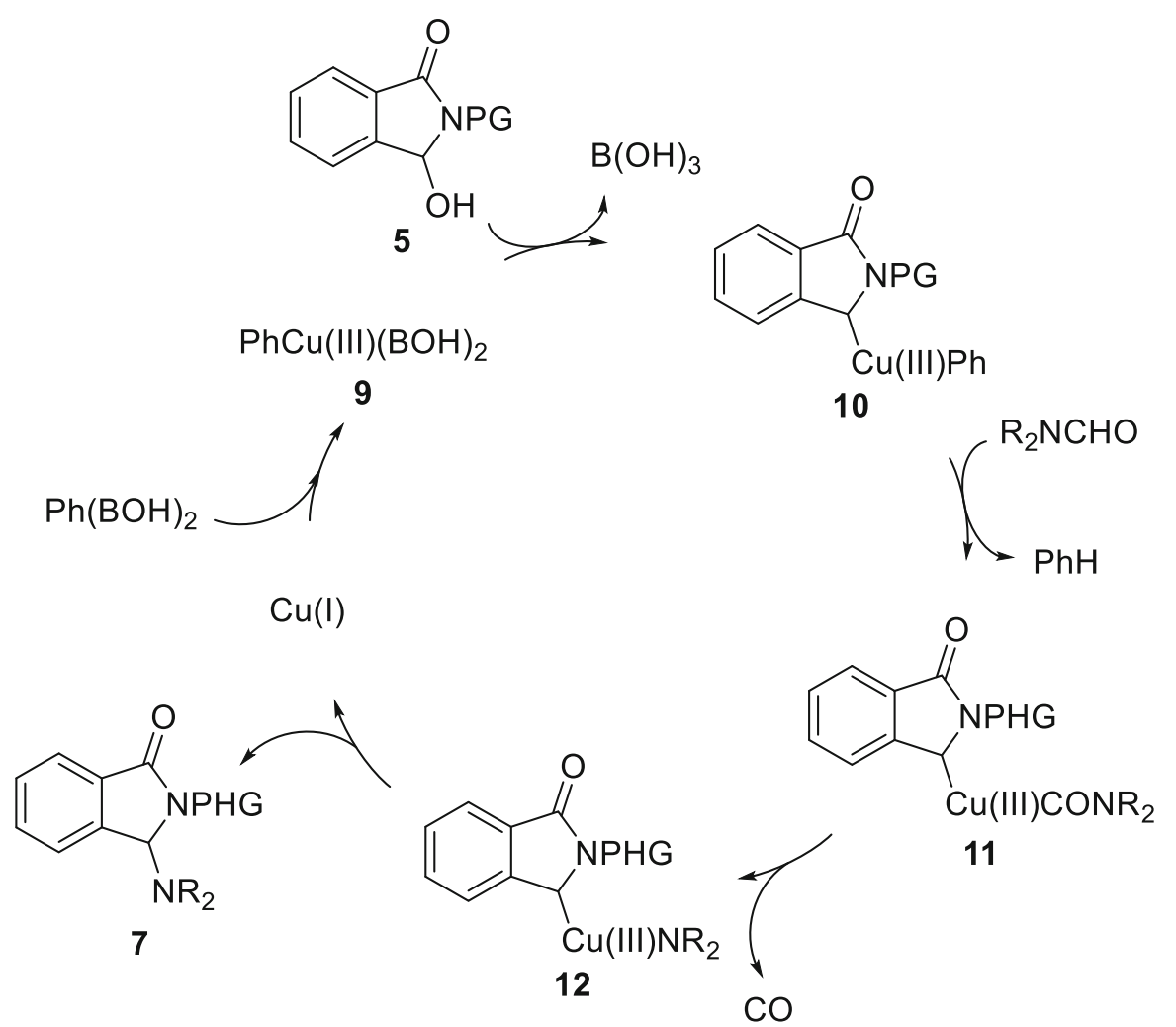

Scheme 3. Proposed mechanism for the copper and phenylboronic acid mediated deformylative amination of isoindolinones.

place; instead it goes through $\mathrm{C}-\mathrm{N}$ bond formation (Scheme 3).

\section{Conclusions}

In conclusion, we have demonstrated an unprecedented $\mathrm{Cu}(\mathrm{I})$ catalyzed and phenylboronic acid-mediated transformation of isoindolinone-3-ols into $\mathrm{C}(3)$ substituted amino isoindolinones. The reaction goes through a deformylative $\mathrm{C}-\mathrm{N}$ coupling. We have demonstrated that DMF is a synthetic equivalent of foul smelling, toxic and gaseous $N, N$-dimethylamine. The transformation has wide scope and can be applied for the synthesis of a combinatorial library of medicinally relevant molecules.

\section{Supplementary Information (SI)}

Characterization data, details of the NMR structural determination of all new compounds and copies of ${ }^{1} \mathrm{H},{ }^{13} \mathrm{C}$ NMR, DEPT-135 and HRMS spectra for all the compounds are given in the supplementary information. Supplementary information is available at www.ias.ac.in/chemsci.

\section{Acknowledgements}

H S P R thanks UGC, UGC-SAP, and DST-FIST for financial assistance and instrumental facilities. J P thanks PU and UGC,
AVR thanks CSIR, and S.K thanks PU for fellowships. We thank CIF, Pondicherry University for recording some of the spectra.

\section{References}

1. (a) Review: Speck K and Magauer T 2013 The chemistry of isoindole natural products Beilstein J. Org. Chem. 9 2048; (b) Scherlach K, Schuemann J, Dahse H M and Hertweck C 2010 Aspernidine A and B, prenylated isoindolinone alkaloids from the model fungus aspergillus nidulans J. Antibiot. 63 375; (c) Chen J, Huang P Q and Queneau Y 2009 Enantioselective synthesis of the $\mathrm{R}$-enantiomer of the feeding deterrent (S)-ypaoamide $J$. Org. Chem. 74 7457; (d) Subbarayappa A and Patoliya P U 2009 An efficient method for the synthesis of 2, 3dihydro- $1 H$-isoindoles Indian J. Chem., Sect. B 48 545; (e) Lamblin M, Couture A, Deniau E and Grandclaudon P 2007 A concise first total synthesis of narceine imide Org. Biomol. Chem. 5 1466; (f) Belliotti T R, Brink WA, Kesten S R, Rubin J R, Wustrow D J, Zoski K T, Whetzel S Z, Corbin A E, Pugsley T A, Heffner T G and Wise L D 1998 Isoindolinone enantiomers having affinity for the dopamine D 4 receptor Bioorg. Med. Chem. Lett. 8 1499

2. (a) Review: Chen H P and Liu J K 2017 Secondary Metabolites from Higher Fungi In Progress in the Chemistry of Organic Natural Products A D Kinghorn, 
H Falk, S Gibbons and J Kobayash (Eds.) (Springer International Publishing) p.106; (b) Yin Y, Fu Q, Wu W, Cai M, Zhou X and Zhang Y 2017 Producing novel fibrinolytic isoindolinone derivatives in marine fungus stachybotrys longispora FG216 by the rational supply of amino compounds according to its biosynthesis pathway Mar. Drugs 15214

3. Zhao J, Liu J, Shen Y, Tan Z, Zhang M, Chen R, Zhao J, Zhang D, Yu L and Dai J 2017 Stachybotrysams A$\mathrm{E}$, prenylated isoindolinone derivatives with anti-HIV activity from the fungus Stachybotrys chartarum Phytochemistry Lett. 20289

4. (a) Li E, Jiang L, Guo L, Zhang H and Che Y 2008 Pestalachlorides A-C, antifungal metabolites from the plant endophytic fungus pestalotiopsis adusta Bioorg. Med. Chem. 16 7894; (b) Slavov N, Cvengroš J, Neudörfl J $\mathrm{M}$ and Schmalz H G 2010 Total synthesis of the marine antibiotic pestalone and its surprisingly facile conversion into pestalalactone and pestalachloride A Angew. Chem., Int. Ed. 497588

5. (a) Reck B and Spiteller P 2015 Synthesis of secondary metabolites from higher fungi Synthesis 47 2885; (b) Di Mola A, Palombi L and Massa A 2014 An overview on asymmetric synthesis of 3-substituted indolinones Targets Heterocycl. Syst. 18113

6. Armoiry X, Aulagner G and Facon T 2008 Lenalidomide in the treatment of multiple myeloma: a review J. Clin. Pharm. Ther. 33219

7. Kondo T, Yoshida K, Yoshimura Y and Tanayama S 1995 Enantioselective pharmacokinetics in animals of pazinaclone, a new isoindoline anxiolytic, and its active metabolite Biopharm. Drug Dispos. 16755

8. For recent literature on synthesis of N(2), C(3)substituted isoindolinones, see (a) Bedford R B, Bowen J G and Méndez-Gálvez C 2017 Isoindolinones via coppercatalyzedintramolecular benzylic $\mathrm{C}-\mathrm{H}$ sulfamidation $J$. Org. Chem. 82 1719; (b) Miura H, Terajima S, Tsutsui $\mathrm{K}$ and Shishido T 2017 Ruthenium-catalyzed addition of aromatic amides to internalalkynes and subsequent intramolecular cyclization for the atom-economical synthesis of isoindolinones J. Org. Chem. 82 1231; (c) Sun L, Liu P, Wang J, Lu P and Wang Y 2017 Preparation of spiro [indene-1, 1' -isoindolin]-3'-ones via sulfuricAcidpromoted cascade cyclization J. Org. Chem. 82 8407; (d) Alonso C, Gonzalez M, Palacios F and Rubiales G 2017 Study of the hetero-[4+2]-cycloaddition reaction of aldimines and alkynes. Synthesis of 1, 5naphthyridines and isoindolonederivatives J. Org. Chem. 82 6379; (e) Folgueiras-Amador A A, Philipps K, Guilbaud S, Poelakker J and Wirth T 2017 An easyto-machine electrochemical flow microreactor:efficient synthesis of isoindolinone and flow functionalization Angew. Chem. Int. Ed. 56 15446; (f) Wu X, Wang B, Zhou Y and Liu H 2017 Propargyl alcohols as onecarbon synthons:redox-neutral rhodium (III)-catalyzed $\mathrm{C}-\mathrm{H}$ Bond activation for thesynthesis of isoindolinones bearing a quaternary carbon Org. Lett. 19 1294; (g) Yu L, Huang H, Chen X, Hu L, Yu Y and Tan Z 2017 Efficient syntheses of 3-hydroxyimino-1-isoindolinones and 3-methylene-1-isoindolinones via $\mathrm{Cu}$-promoted $\mathrm{C}-$ Hactivation-nitroalkylation-intramolecular cyclization tandem processes Chem. Commun. 53 4597; (h) Xu Y,
Wang F, Yu S and Li X 2017 Rhodium (III)-catalyzed selectiveaccess to isoindolinones via formal [4+ 1] annulation of arylamidesand propargyl alcohols Chin. J. Catal. 381390

9. Di Mola A, Palombi L and Massa A 2016 An overview on asymmetric synthesis of 3-substituted isoindolinones ChemInform 47113

10. (a) Rao H S P and Rao A V B 2014 Copper-Catalyzed $\mathrm{C}\left(\mathrm{sp}^{3}\right)-\mathrm{C}\left(\mathrm{sp}^{2}\right)$ Cross-coupling: synthesis of 4-aryl-2alkylamino-3-nitro-4H-chromenes Eur. J. Org. Chem. 17 3646; (b) Rao H S P and Rao A V B 2016 Coppermediated arylation with arylboronic acids: Facile and modular synthesis of triarylmethanes Beilstein J. Org. Chem. 12 496; (c) Rao H S P, Rao A V B, Sivakumar S 2015 Copper (II) bromide-catalyzed C-C/C-N bondforming reactions: synthesis of pyrrole-incorporated triarylmethanes Synth. Commun. 452712

11. Rao H S P and Rao A V B 2015 Copper-catalyzed $\mathrm{C}\left(\mathrm{sp}^{3}\right)-\mathrm{OH}$ cleavage with concomitant $\mathrm{C}-\mathrm{C}$ coupling: synthesis of 3-substituted isoindolinones J. Org. Chem. 801506

12. (a) Review: Maaliki C, Thiery E and Thibonnet J 2017 Emergence of copper-mediated formation of CC bonds Eur. J. Org. Chem. 209; (b) Review: Allen S E, Walvoord R R, Padilla-Salinas R and Kozlowski M C 2013 Aerobic copper-catalyzed organic reactions Chem. Rev. 113 6234; (c) Sahoo H, Mukherjee S, Grandhi G S, Selvakumar J and Baidya M 2017 Copper catalyzed $\mathrm{C}-\mathrm{N}$ cross-coupling reaction of aryl boronic acids at room temperature through chelation assistance J. Org. Chem. 82 2764; (d) Mandal P S and Kumar A V 2016 Copper-catalyzed imino C-N bond formation with aryl boronic acids under aerobic conditions Synlett. 27 1408; (e) Petrassi H M, Sharpless K B and Kelly J W 2001 The copper-mediated cross-coupling of phenylboronic acids and N-hydroxyphthalimide at room temperature: synthesis of aryloxyamines Org. Lett. 3 139

13. (a) Capobianco A, Di Mola A, Intintoli V, Massa A, Capaccio V, Roiser L, Waser M and Palombi L 2016 Asymmetric tandem hemiaminal-heterocyclization-azamannich reaction of 2-formylbenzonitriles and amines using chiral phase transfer catalysis: an experimental and theoretical study $R S C A d v .6$ 31861; (b) Palombi L, Di Mola A and Massa A 2015 Quick and easy access to Nmannich bases of 1-isoindolinones by catalytic electro activation of primary and secondary amines and tandem reaction with 2-formylbenzonitriles New J. Chem. 39 81

14. (a) Review: Zhang C, Tang C and Jiao N 2012 Recent advances in copper-catalyzed dehydrogenative functionalization via a single electron transfer (SET) process Chem. Soc. Rev. 41 3464; (b) Huang X, Wang J, Ni Z, Wang S and Pan Y 2014 Copper-mediated S-N formation via an oxygen-activated radical process: a new synthesis method for sulfonamides Chem. Commun. 50 4582; (c) Kumar, G S, Maheswari C U, Kumar R A, Kantam M L and Reddy K R 2011 Copper-catalyzed oxidative $\mathrm{C}-\mathrm{O}$ coupling by direct $\mathrm{C}-\mathrm{H}$ bond activation of formamides: Synthesis of enol carbamates and 2-carbonyl-substituted phenol carbamates Angew. Chem., Int. Ed. $\mathbf{5 0} 11748$ 
15. Armarego W L F and Chai C L L 2003 Purification of Laboratory Chemicals $5^{\text {th }}$ Ed. (Oxford, UK: Elsevier)

16. See Supplementary Information
17. Gerber M J, Gorczynski R J and Roden R L 2007 Antihypertensive Therapy PCT Int. Appl. WO 2007098387 A2 